\title{
Realistic modeling of ion cloud motion in a Fourier transform ion cyclotron resonance cell by use of a particle-in-cell approach
}

\author{
Eugene N. Nikolaev ${ }^{\text {* }}$, Ron M. A. Heeren ${ }^{3}$, Alexander M. Popov², Alexander V. Pozdneev ${ }^{2}$ \\ and Konstantin S. Chingin ${ }^{1}$
}

${ }^{1}$ The Institute for Energy Problems of Chemical Physics, Russian Academy of Sciences, Leninskij pr.38, k.2, Moscow, Russia 119334

${ }^{2}$ Department of Computer Sciences, Moscow State University, Moscow, Russia

${ }^{3}$ FOM Institute for Atomic and Molecular Physics, Amsterdam, The Netherlands

Received 12 September 2006; Revised 3 August 2007; Accepted 20 August 2007

\begin{abstract}
Using a 'Particle-In-Cell' approach taken from plasma physics we have developed a new threedimensional (3D) parallel computer code that today yields the highest possible accuracy of ion trajectory calculations in electromagnetic fields. This approach incorporates coulombic ion-ion and ion-image charge interactions into the calculation. The accuracy is achieved through the implementation of an improved algorithm (the so-called Boris algorithm) that mathematically eliminates cyclotron motion in a magnetic field from digital equations for ion motion dynamics. It facilitates the calculation of the cyclotron motion without numerical errors. At every time-step in the simulation the electric potential inside the cell is calculated by direct solution of Poisson's equation. Calculations are performed on a computational grid with up to $128 \times 128 \times 128$ nodes using a fast Fourier transform algorithm. The ion populations in these simulations ranged from 1000 up to 1000000 ions. A maximum of 3000000 time-steps were employed in the ion trajectory calculations. This corresponds to an experimental detection time-scale of seconds. In addition to the ion trajectories integral time-domain signals and mass spectra were calculated. The phenomena observed include phase locking of particular $\mathrm{m} / \mathrm{z}$ ions (high-resolution regime) inside larger ion clouds. A focus was placed on behavior of a cloud of ions of a single $\mathrm{m} / \mathrm{z}$ value to understand the nature of Fourier transform ion cyclotron resonance (FTICR) resolution and mass accuracy in selected ion mode detection. The behavior of two and three ion clouds of different but close $\mathrm{m} / \mathrm{z}$ was investigated as well. Peak coalescence effects were observed in both cases. Very complicated ion cloud dynamics in the case of three ion clouds was demonstrated. It was found that magnetic field does not influence phase locking for a cloud of ions of a single $\mathrm{m} / \mathrm{z}$. The ion cloud evolution time-scale is inversely proportional to magnetic field. The number of ions needed for peak coalescence depends quadratically on the magnetic field. Copyright (C) 2007 John Wiley \& Sons, Ltd.
\end{abstract}

The leading role Fourier transform ion cyclotron resonance mass spectrometry (FTICR-MS) plays in biological mass spectrometry is directly related to its unsurpassed resolution and mass accuracy combined with the possibility of utilizing all known ionization and fragmentation methods. Further improvement of mass accuracy will be greatly facilitated by a more detailed understanding of the motion of ions during ion introduction into the FTICR cell, excitation of their cyclotron motion, fragmentation and detection of induced signal. Starting from the early days of FTICR-MS the theory of ion motion in the ICR cell was an important research topic in this field. The dynamics of single particles inside the FTICR trap was analyzed by many groups in both the FTICR and physics communities. ${ }^{1-8}$ The FTICR community has

${ }^{*}$ Correspondence to: E. N. Nikolaev, The Institute for Energy Problems of Chemical Physics, Russian Academy of Sciences, Leninskij pr.38, k.2, Moscow, Russia 119334.

E-mail: ennikolaev@rambler.ru been interested mainly in obtaining a calibration formula, which connects measured frequency with ion mass. ${ }^{9}$

The early studies investigated the effects of various external fields and ion-neutral collisions on single particle trajectories. ${ }^{10-16}$ The availability of the SIMION program ${ }^{17}$ simplified the task of understanding different aspects of single ion motion in cells of arbitrary geometry. This PC-based ion trajectory program is able to show single ion trajectories in different kinds of ICR cells with relatively high accuracy. The simulated time-scales are comparable with the time-scales of typical FTICR experiments. Because of the lack of appropriate ion-ion interaction routines SIMION fails in the study of the influence of coulombic interaction and image charge on ion motion. FTICR-MS generally deals with ion clouds containing at least hundreds of ions rather than single

(4ILEY

InterScience 
particles. As a result the trajectory obtained from single ion simulations does not provide a realistic insight into the ion trajectories in the presence of other ions. In accurate mass measurements using ion traps single ions of different $m / z$ are introduced into the cell consecutively. Even these precautions are not enough to avoid fully the ion-ion interaction problem since ion-image charge interaction still exists and causes the diocotron ${ }^{\mathrm{a}}$ motion $^{18}$ thus disturbing cyclotron motion frequency. This effect introduces mass errors of the order of $10^{-8}$ to $10^{-9}$ crucial to absolute mass measurements. ${ }^{19}$ The relative measurement errors of the order of $10^{-7}$ limit more practical FTICR experiments. At this level of accuracy ion-ion and ion-image charge interactions play a crucial role with just hundreds of ions trapped in the cell. No analytical solutions exist to date to evaluate these mass measurement errors. Several groups have analyzed the ion motion using different approximations to calculate the electric field in the cell and treat ion-ion interaction and a number of models have been proposed ${ }^{3,10,20-23}$ to evaluate cyclotron frequency shifts induced by space charge. They are based on determining an average radial force arising from static charge distribution of the ion cloud during detection. Nikolaev's group ${ }^{24,25}$ developed a two-dimensional (2D) model for an infinitely long cylindrical cell. This model yields an analytical expression for frequency shifts caused by ion-ion and ion-image charge interactions in a single ion approximation. This simple model predicts correctly the magnitude of the experimentally observed frequency shifts when the ion number density is low and cyclotron frequencies of different species differ significantly. It also provides a simple approach to the treatment of ICR signal harmonics. ${ }^{26}$ Few simulations have been published on large populations of coulombically interacting particles in ICR mass spectrometers. The role of ion-ion interaction and image charge was first described by Talroze and Nikolaev ${ }^{19}$ in connection with mass difference measurements of the ${ }^{3} \mathrm{He} / \mathrm{T}$ doublet. ${ }^{27}$ Later on, Peurrrung et al. ${ }^{28}$ developed a charge plasma approach to tackle the problem and analyzed cases of high charge density ion clouds. Simple 2D simulation programs have been used by different groups to investigate various phenomena related to coulombic interactions in FTICR. ${ }^{3,4,29-31}$ The first attempt to implement coulombic interaction in realistic three-dimensional (3D) calculations of simulations involving a relatively small number of interacting particles was undertaken by the groups of Inoue and Nikolaev. They developed a 3D parallel code to integrate the equations of motion of up to 1024 interacting particles for 50000 time-steps. $^{32,33}$ They used a parallel processing approach implemented on a supercomputer with up to 1024 processors to calculate the time and space evolution of a single $\mathrm{m} / \mathrm{z}$ ion cloud. These simulations took into account ion-ion interaction but neglected image charge effects. They determined the form of an ICR peak and mass error as a function of initial conditions such as ion cloud shape and the number of ions in the cloud. A different approach to this problem was used by Dale Mitchell, who applied a 3D many particles simulator using a particle-in-cell algorithm devel-

${ }^{\text {a }}$ The motion in the magnetic field caused by image charge attraction force. oped in plasma physics. He applied this approach to the problem of cyclotron frequency locking (peak coalescence) of two closely spaced masses under high space charge conditions. ${ }^{1,2,4,34-37}$ His calculations were initially performed in 2D Cartesian and cylindrical confinement geometries utilizing the Monte Carlo algorithm to treat ion-neutral collisions. ${ }^{5,38-40}$ As was shown by Mitchell, at low magnetic field, high number density, or closely spaced cyclotron frequency separation, the ion motion dynamics becomes considerably more complicated than just a frequency shift. Later on ${ }^{6}$ he carried out a more realistic 3D many particles simulation of trapped ion clouds incorporating space charge effects (coulombic and image charge interactions) for a sufficiently large ion population. All major aspects of the experiment including trap boundary, applied potentials, magnetic field, and neutral bath gas were included in this model. He calculated the evolution of the shape of two coulombically interacting ion clouds during the detection period as a function of the number of ions in the cloud. This approach permits the incorporation of coulombic ion-ion and ion-image charge interactions for an experimentally realistic number of ions in the cell. The goal of the present paper is to extend this particle-in-cell approach to allow for the simulation of many particles dynamics inside the FTICR cell on the basis of improved computational algorithms and using the most powerful computers available at this time. The present work describes a new implementation of a 3D particle-in-cell code. The code can model a complete FTICR experiment with up to $10^{6}$ coulombically interacting ions in the ICR cell for 3000000 time-steps. This new code allows for the simulation of almost any practical FTICR sequence and experimental conditions. In addition it can easily be extended to model other types of devices relevant to modern high-performance mass spectrometric studies.

\section{METHOD}

In Fourier transform ion cyclotron resonance mass spectrometry (FTICR-MS) a strong magnetic field parallel to the symmetry axis of an ICR trap (Penning trap) confines the ions radially. ${ }^{41-45}$ Trapping potentials applied to trapping electrodes of the cell ${ }^{46-49}$ simultaneously confine the ions in a direction parallel to the magnetic field.

A typical sequence of an FTICR-MS measurement consists of successive steps of ion accumulation in the ICR trap, damping ion axial motion, excitation of the cyclotron motion, and signal detection by measuring the oscillating image charge induced on the detection plates. A radio-frequency (RF) voltage applied to a set of excitation plates (positioned parallel to the magnetic field and creating an RF electric field perpendicular to the magnetic field) excites the cyclotron mode during the excitation period. The image charge induced on an additional set of trap electrodes is detected as a decaying time-dependent signal during the detection period. A Fourier transform of this time-domain signal yields the cyclotron frequency spectrum containing the cyclotron frequency of the ions.

When an ensemble of particles is stored in the ICR cell, every particle experiences the Lorentz force caused by the magnetic field and trapping electric fields as well as 
coulombic forces resulting from the presence of the other ions in the cell and from the image charges induced in the cell electrodes by the ions present inside the cell. In principle it is possible to calculate exactly the total coulombic force acting on every individual particle using the Green function ${ }^{b}$ approach. This method for direct force calculation is called the particle-particle method. Analytical expressions for the Green functions are known for simple cubic or cylindrical trap geometries. Unfortunately, the time needed to calculate the total force acting on every particle in the ensemble is too long for a reasonable number of particles in the cloud even for the most powerful computers. This is a direct result of the fact that even for these simple geometries these functions are represented by a series with an infinite number of terms. Hence, this approach is not practical at the current level of computer development. A truncated particle-particle algorithm, in which the influence of image charge is neglected, could be used without any computational problems to calculate ion dynamics in clouds positioned far from the cell electrodes. The other approach which could be used in the particle-particle method is the principle of reciprocity, ${ }^{50,51}$ applied to ICR by Dunbar ${ }^{52}$ and Grosshans. ${ }^{53}$ It avoids the Green function by computing the potential at a point in the cell produced by the surrounding electrodes, and then obtains the charge induced in detector electrodes by reciprocity-that does not include ion-ion interactions, but converges in a few hundred terms.

The particle-in-cell (or particle-in-mesh) algorithms developed in plasma physics seem to be the most adequate solution to the problem. Classical electrodynamics dictates that to find the force, $\mathbf{F}\left(\mathbf{r}_{i}\right)=-q_{i} g r a d \varphi\left(\mathbf{r}_{i}\right)$, acting on individual ion $i$ at position $\mathbf{r}_{i}$ from the other ions and trap electrodes we should solve the Poisson equation, $\Delta \varphi=-\rho / \varepsilon_{0}$. Here, the charge density $\rho$ is determined by the distribution of ions inside the cell for the field potential $\varphi$ with Dirichlet boundary conditions (fixed potentials on the trap electrodes). By solving the Poisson equation we are accounting for image charge as well. To establish the charge density $\rho$ at any arbitrary point inside the trap we should specify the physically small volume $v$, calculate the number of ions $n$ inside this volume, and divide $n$ by $v: \rho=n / v$. In the particle-in-cell method the whole space where the Poisson equation should be solved is subdivided into small cubic volumes $v$ on a regular mesh with equidistant nodes and $\rho$ is determined at every node of the mesh by a spatial interpolation algorithm.

\section{Particle-in-cell algorithm}

The particle-in-cell (PIC) algorithm ${ }^{38-40}$ consists of numerous consecutive time-steps. At each time-step the field is calculated in the whole volume of interest and ion cloud motion in this field is simulated. In the PIC algorithm the whole space where the ion dynamics is investigated is subdivided into a number of equal volume cubic cells forming a regular 3D mesh. The charge of every individual particle is interpolated from the particle's position inside the cell onto the discrete grid points on the computational mesh

${ }^{b}$ The Green function $G(\mathbf{r}, \mathbf{R})$ is a potential induced at some point $\mathbf{r}$ of the space inside the trap by a singly charged ion positioned inside the trap at point $\mathbf{R}$.

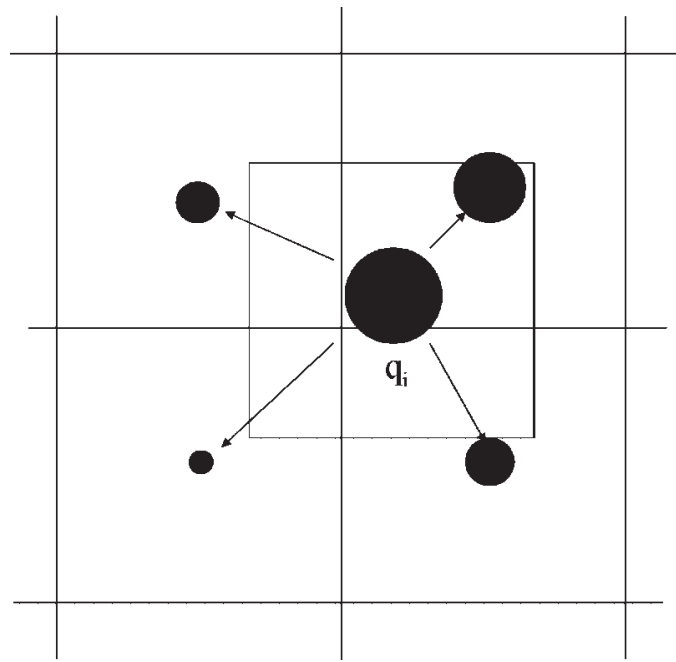

Figure 1. Weighting algorithm principle. The ion density distribution is determined at every node of the computational mesh by interpolating the charge at a particle position to the charge density at grid points. The same scheme is used to interpolate electric field at grid points to particle positions.

by using a 3D weighting algorithm. This weighting algorithm used volume weighting in most of the calculations reported in this paper (see Fig. 1 and Appendix 1). In the simplest mode of this algorithm the ion density distribution is determined at every node of the mesh by dividing the total charge extrapolated to this particular node by the volume of the elementary cube of the mesh. Using a direct fast Fourier transform (FFT) Poisson solver with the trap boundary conditions, corresponding to the direct current (DC) and RF potentials on the trap electrodes, this charge density on the grid points was converted into potentials at these points and electric fields from the spatial derivatives of the potentials. The electric field at an individual ion's position is calculated by interpolation of the electric fields from the nearest grid points using the same weighting algorithms as used for charge projection to the grid points. The mathematical description of the whole procedure of solving the Poisson equation by the FFT method is described in Appendix 2.

The particle positions and velocities of the next time-step are calculated using the Boris integrator ${ }^{38,54}$ (see Appendix 3). The integrator algorithm permits the separation of the ion motion into motion in pure electric and pure magnetic fields, and so calculates the motion in the magnetic field during each time-step thus analytically avoiding any computational errors in these calculations. Ion-neutral collisions are implemented by the Monte Carlo method using an elastic hard sphere and Langevin approximation (see Appendix 4). (In many of the calculations for which results are presented in this paper, ion-neutral collisions were not used.) The calculation procedure is repeated for the predetermined number of time-steps. The image charge on the trap electrodes is calculated by integration of the charge on the internal surfaces of the detection electrodes, the density of which is proportional to the normal component of the electric field on the surface. The detected ICR signal is subsequently determined as the first-order derivative of the total charge on one of the detecting electrodes. 


\section{The model}

In our current model we used a cell of cubic geometry with dimensions $1 \times 1 \times 1$ inch. This geometry simplifies and accelerates significantly the field calculation by the Poisson solver as it permits usage of an FFT algorithm to calculate the field. DC voltages are continuously applied to the trapping electrodes $(+1 \mathrm{~V}$ to both) and the $\mathrm{RF}$ is applied to the excitation electrodes during the excitation period only. The detection electrodes stay grounded at all times.

\section{The choice of the cell size}

In the particle-in-cell (PIC) method the interaction between two ions is in fact substituted by the interaction of one of the ions with eight charges on the mesh nodes nearest to the second ion. The accuracy of this representation is greater as the distance between the two ions becomes larger. As a result, the method becomes more accurate if the ion density (the number of ions per cell) or the mesh cell size is decreased (see Appendix 5). Decreasing the cell size and increasing the number of mesh nodes is computationally costly because the number of operations is proportional to the number of nodes, which increases cubically with reversed cell linear size. We used two types of mesh with $64 \times 64 \times 64$ and $128 \times 128 \times 128$ nodes, respectively. For the latter case this corresponds to an average of one ion per cell for $10^{6}$ ions.

\section{RESULTS}

\section{Single $m / z$ ion cloud}

It is known from experiments that the initial distribution of the ion cloud prior to excitation plays a crucial role in the formation of the ion cloud during excitation and its subsequent behavior. In an experiment it depends strongly on the ion accumulation method used and the relaxation period prior to excitation and detection. In the simple case where ion formation is realized inside the ICR trap by electron impact or photoionization with beams centered in the trap, ions are created on the axis with random distribution along the trap (assuming that the ionization time is much longer than the ion axial oscillation period). If we introduce a time delay between the ionization and excitation events, ions will lose their oscillatory kinetic energy in collisions with neutrals. Their spatial distribution along the $z$-axis narrows and the dimension of the cloud in the radial direction increases.

For externally formed ions there are at least two trapping modes in use. In one of these modes ions are accumulated in an intermediate multipole trap and subsequently ejected into the ICR cell, where they are trapped. In the other mode semi-continuous gas-assisted trapping is used. In this paper we have not analyzed processes in the ion cloud during ion formation in the cell or ion introduction into the cell. We have investigated the initial spatial distribution and changed it from compact cylindrical to elongated cylindrical and elliptical shapes with different aspect ratios (from a coin-like form to a rod-like form). Owing to the relaxation period between ion introduction and the excitation event in all FTICR-MS experiments, we have chosen to employ a constant ion density (at the beginning) and a spatial distribution arising as a result of the collisional relaxation

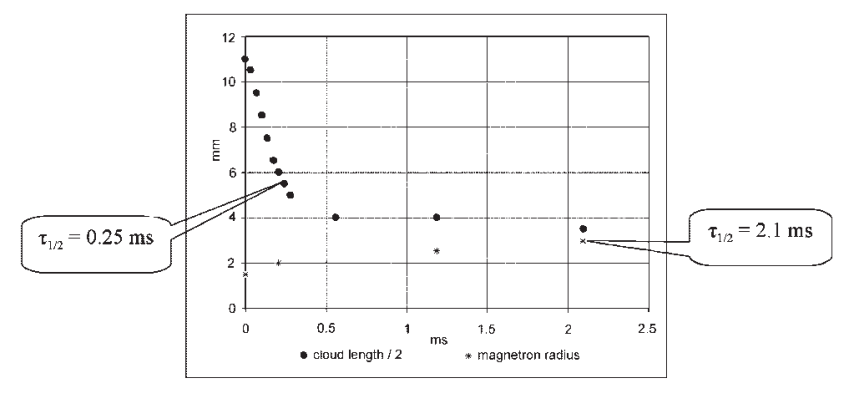

$\tau_{1 / 2}-$ half (twice)-value period

Figure 2. The evolution of an ion cloud of single mass $(\mathrm{m} / \mathrm{z}$ 100.0) ions started from a randomly filled elongated cylinder. Helium was used as a collisional gas at a pressure of $1.5 \mathrm{~m}$ Torr. One can see that the axial relaxation is an order of magnitude faster than the radial spreading of the cloud.

of the initial distributions of different aspect ratios. Initial velocity distributions are always Maxwellian distributions. In the cases where no collision gas is used for ion relaxation we introduced a time lag between initial ion distribution formation and the start of the RF-excitation for the ion cloud to relax by ion-ion collisions. The evolution of the ion cloud from a randomly filled elongated cylinder was simulated and results are presented in Fig. 2. Helium was used as a collisional gas at a pressure of $1.5 \mathrm{mTorr}$. Unreasonably high helium gas pressure was used to observe the effect of ion-neutral collisions in the necessarily short time period limited by the computational speed of the computer. We can see from Fig. 2 that axial relaxation is approximately an order of magnitude faster than the radial spreading of the cloud.

\section{Excitation}

The choice of excitation time and waveform is important because it influences strongly the excitation radius, the coherence of the ion package and their distribution. It is well known that in a cell with cubic geometry the excitation field is very inhomogeneous. It has a maximum value in the cell center and decreases to zero near the trapping electrodes. As a result, ions with different axial oscillation amplitudes are experiencing different excitation field strengths during a single period of axial motion. In Figure 3 the shape of the ion

xy

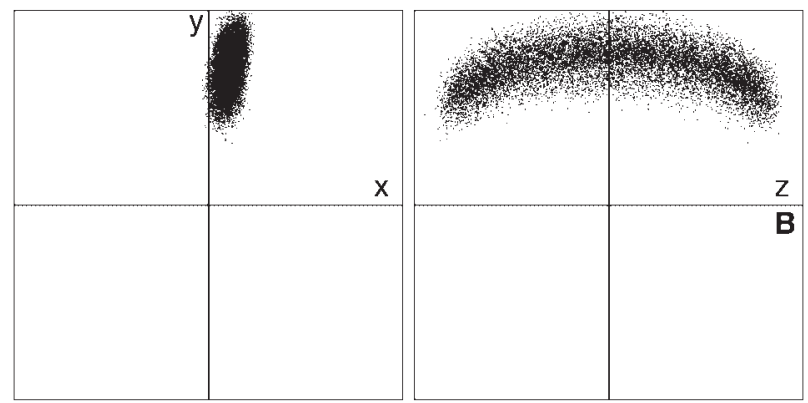

Figure 3. Snapshots in $x y$ (left) and $z y$ (right) view just after the excitation period. Z-elongated $\mathrm{m} / \mathrm{z} 100.0$ ion cloud was excited by applying pure sine RF voltage for $70 \mu \mathrm{s}$ $\left(\mathrm{V}_{\mathrm{pp}}=10 \mathrm{~V}\right)$. Inhomogeneity of the cubic trap excitation field results in the rainbow-shaped ion cloud. 
cloud after excitation is presented when a random initial axial distribution is used. In this figure and in all figures showing ion clouds, the boundaries of the frames coincide with the positions of the excitation and detection electrodes. Experimentally, it is practically nearly impossible to form ion clouds in the cell with similar axial localizations. Even when the ion cloud formation time is shorter than the axial oscillation period $\mathrm{T}_{\text {axial }}$ (e.g. for very short electron ionization or photoionization and gated trapping), the ions will axially oscillate with different amplitudes. The excitation radius will thus depend on the phase shift between excitation pulse and phase of ion oscillation. As a consequence, it is impossible to excite ions to the same radius even if $t_{\text {excite }}$ is much larger than $\mathrm{T}_{\text {axial. }}$. If the relaxation time is large enough for ions to decrease their axial oscillation amplitudes it becomes possible to make the cyclotron motion radius more consistent. Under standard experimental conditions using
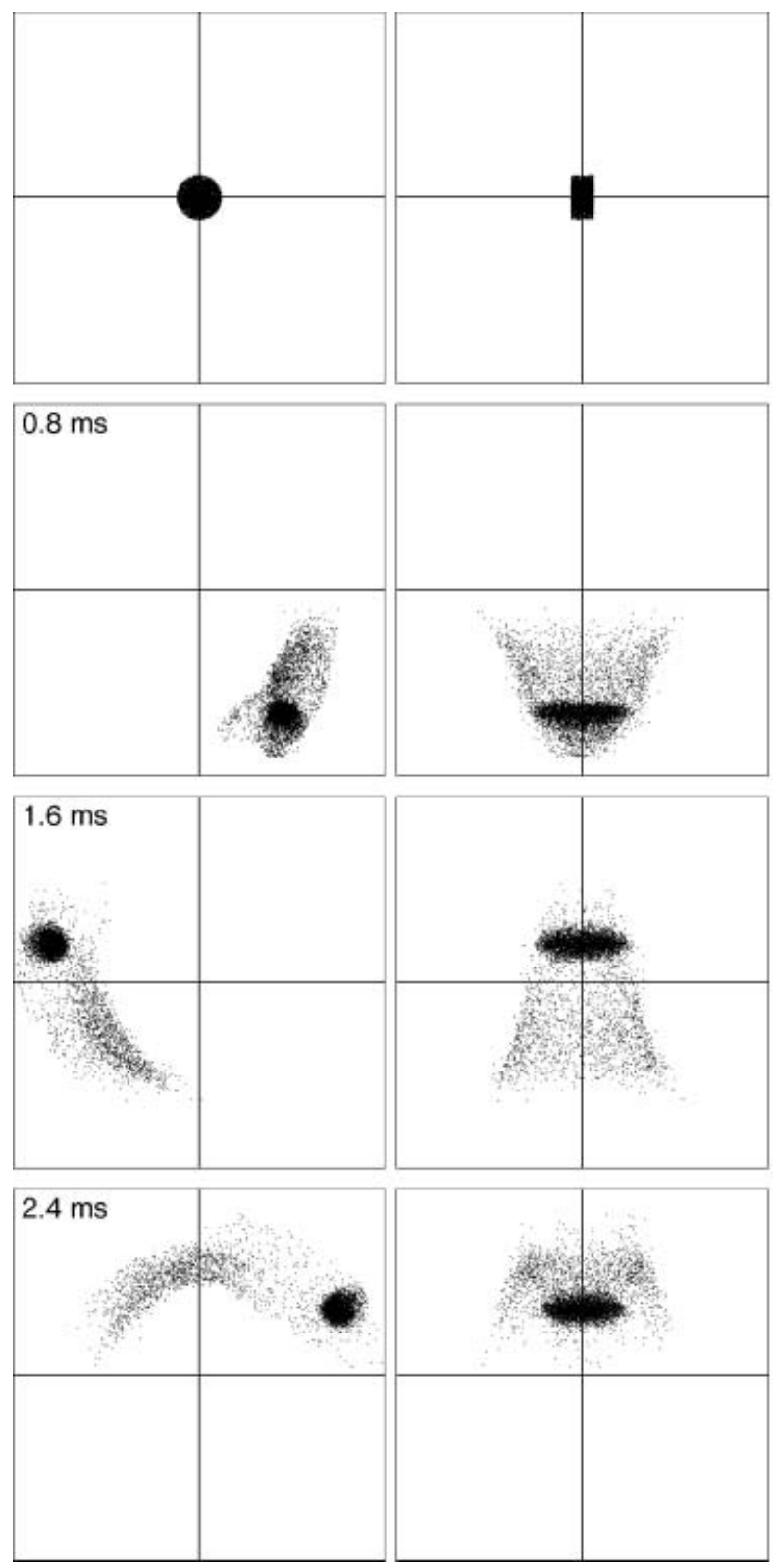

Figure 4. Dynamics of the initially $z$-stressed ion cloud (50000 ions) during the detection period after $70 \mu s$ excitation phase. Snapshots of ion $x y\left(1^{\text {st }}\right.$ and $3^{\text {rd }}$ columns) and $z y\left(2^{\text {nd }}\right.$ and $4^{\text {th }}$ columns) positions are shown; the cubic trap boundary is indicated. The phase-locked ions are concentrated outside the cylinder formed by unsynchronized ions.

\section{The role of initial conditions (ion cloud initial form)}

In Figure 4 a series of pictures of the ion cloud at consecutive time-steps $(0-8.8 \mathrm{~ms})$ starting from the beginning of the excitation period are presented using two projections (one
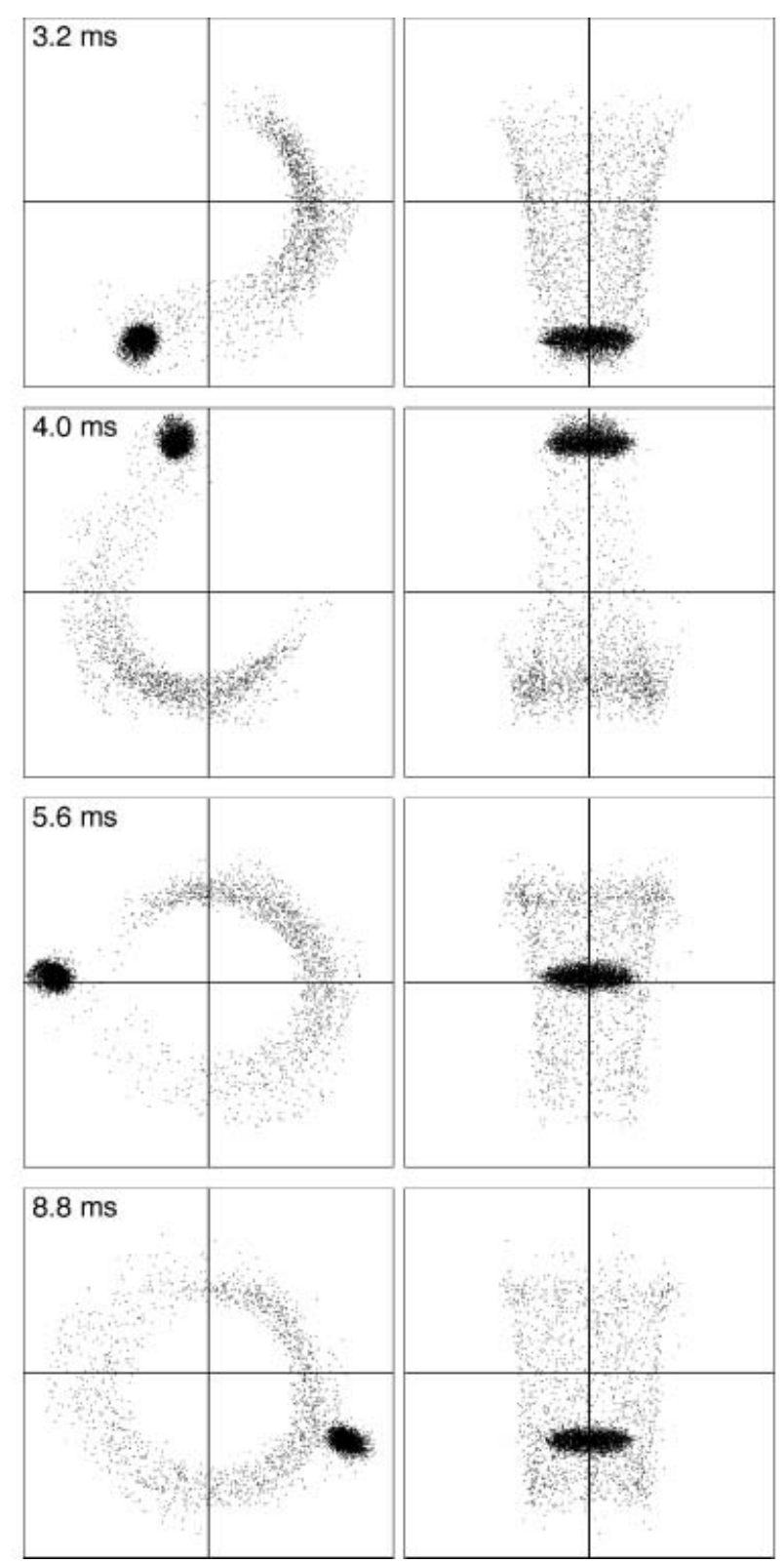

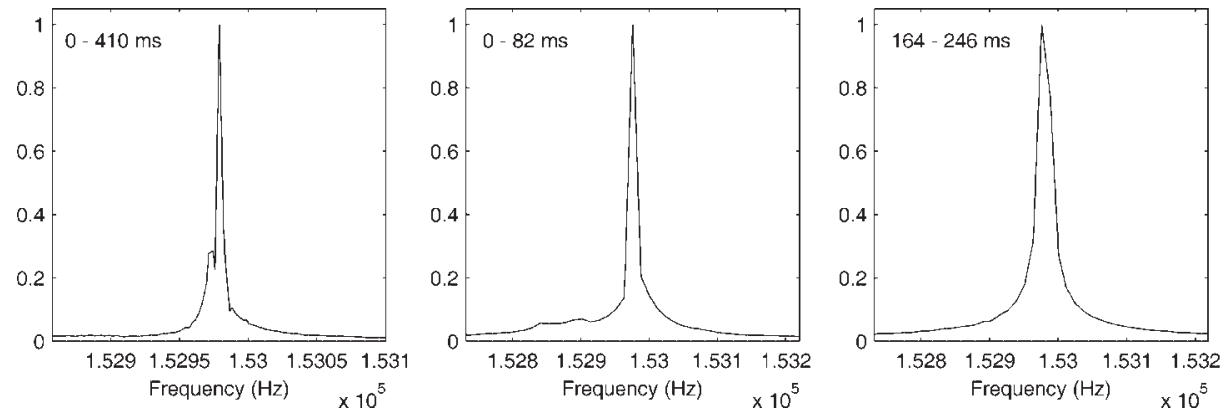

Figure 5. Fourier spectra obtained from different parts of the time-domain signal for the ion cloud evolution presented in Fig. 4.
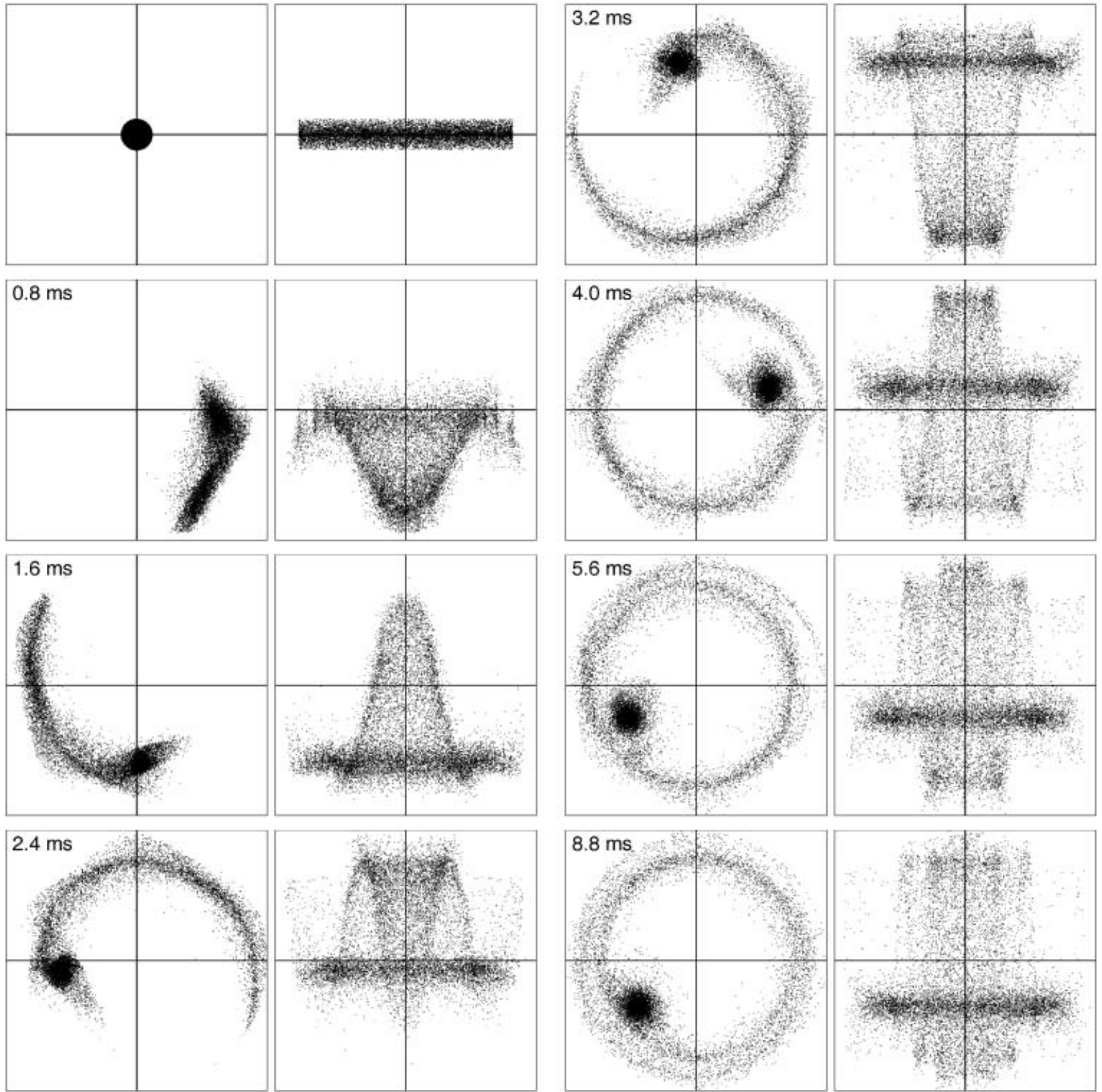

Figure 6. Z-elongated ion cloud evolution (50000 ions) during the detection period after $70 \mu s$ excitation time (snapshots layout is the same as in Fig. 4). The phase-locked ions are encapsulated inside the cylinder formed by unsynchronized ions. 
perpendicular to the magnetic field and one in the plane parallel to the magnetic field). The calculations were performed for 50000 ions using an excitation time of $70 \mu \mathrm{s}$, an RF-excitation amplitude of $10 \mathrm{~V}$ and a magnetic field of $1 \mathrm{~T}$. $^{\mathrm{C}}$ For this particular digital experiment we used the cylindrically shaped initial ion cloud with a cylinder randomly filled by ions with a Maxwellian velocity distribution. The initial geometry of the ion cloud is presented in the first frame. We can call this geometry $z$-stressed because of its compact distribution in the z-direction. A comet formation is clearly observed starting $1 \mathrm{~ms}$ after the beginning of the excitation event. The head of the comet has an elliptical structure and comprises phase-locked ions moving synchronously as a rigid body. After approximately $4 \mathrm{~ms}$ the head is separated from the tail of the comet. The tail loses synchronization and after around $8 \mathrm{~ms}$ forms a cylinder. The comet head is rotating around this cylinder during a relatively long time (of the order of magnitude of $100 \mathrm{~ms}$ ) and sometimes disappears completely. Fourier spectra obtained from different parts of the time-domain signal are presented in Fig. 5. The results of this in silico experiment clearly show three significant features: (i) Comet-shaped ion cloud formation as predicted by earlier research; ${ }^{55}$ (ii) Phase locking of a part of the ion cloud; (iii) Formation of a long-lived structure, in which the ellipsoid of phase-locked ions revolves around the cylinder formed by non-phase-locked ions.

In Fig. 6 the results of the same experiment with different initial ion distributions are presented. In this case we started with an elongated cylinder form of the ion cloud. The behavior of the cloud is very different than described for $z$-stressed geometry in the previous paragraph. We also see comet formation but the phase locking occurs in the tail. The phase-locked ions are encapsulated inside the cylinder formed by other ions. In this particular digital experiment synchronous motion of locked ions survives for a longer time than in the $z$-stressed case. From these two experiments it is evident that the initial form of the ion cloud plays a crucial role in FTICR-MS signal formation. It is clear that peak positions in these two cases will be different for at least two reasons: (i) because in the first case phase-locked ions will experience coulombic forces from the ions forming the internal cylinder and in the second case according to Gauss' law this interaction is absent and (ii) because in the second case the averaged magnetron frequency is higher and effective measured frequency smaller. These two effects have opposite signs and the resulting frequency shift could be of both signs. In both cases (z-stressed and elongated geometry of clouds), the frequency shift depends on the portion of total number of ions redistributed between phase-locked and dephased ensembles making measured frequency time dependent and pick position stochastical.

\footnotetext{
${ }^{\mathrm{c}}$ The choice of a relatively small magnetic field strength not used in current FTICR practice is caused by two reasons: first the possibility to compare results with what Mitchell obtained in his research at $1 \mathrm{~T}$ and, second, to accelerate the computational time, which is inversely proportional to cyclotron frequency and proportional to the magnetic field at the chosen accuracy of the calculations.
}

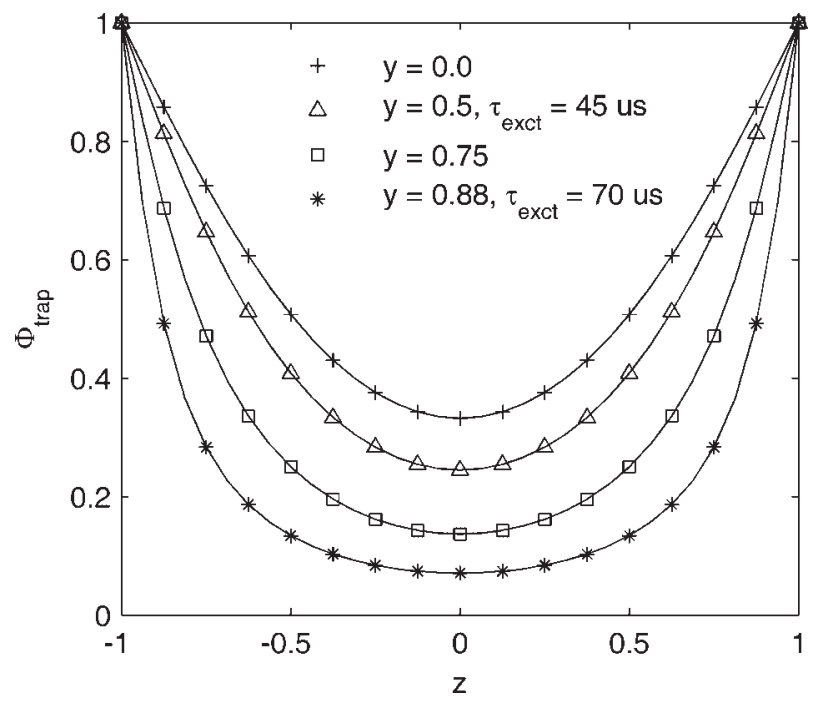

Figure 7. The distribution of the potential along the magnetic field for the cubic cell at different distances from the cell axis in the $x=0$ plane. Profiles for $y=0.5$ and $y=0.88$ correspond to excitation times of $45 \mu \mathrm{s}$ and $70 \mu \mathrm{s}$. The bottom of the potential gets flatter as ions approach the side electrodes during excitation of their cyclotron motion.

This phenomenon will have significant effects on the mass accuracy that can be achieved.

\section{The role of the excitation time}

In Fig. 7 the distribution of the potential along the magnetic field is presented for the cubic cell at different distances from the axis of the cell. These distributions were calculated by solving Laplace's equation for cubic cell geometry used in our modeling. The bottom of the potential well becomes flatter when ions approach the side electrodes during excitation of the cyclotron motion. Consequently, the electric field contribution from the trapping electrode gets smaller and the ions are freer to spread along the $z$-axis at higher cyclotron radius.

At smaller radii ions are more compressed in the $z$-direction and phase locking should be more pronounced. This effect can be seen in Fig. 8, where ion cloud dynamics were followed for 5000, 20000 and 50000 ions using an excitation time of $45 \mu \mathrm{s}$. We do not see any comet formation when 50000 ions are used and almost total phase locking is observed. Even in the case of 5000 ions in the cell phase locking takes place for some of them (see below).

\section{The role of the number of ions in the cloud}

Figures 8 and 9 demonstrate that the number of ions plays a crucial role in phase locking and determines the total time of synchronous ion motion and as a result the mass resolution. In Fig. 9 the dynamics of ion clouds with 5000, 20000 and 50000 ions are presented for an excitation time of $70 \mu \mathrm{s}$.

\section{Ion losses during excitation and detection periods}

In Figure 10 the total number of ions in the cell is presented as a function of the time after the start of the RF-excitation event. It demonstrates significant losses during excitation for high 

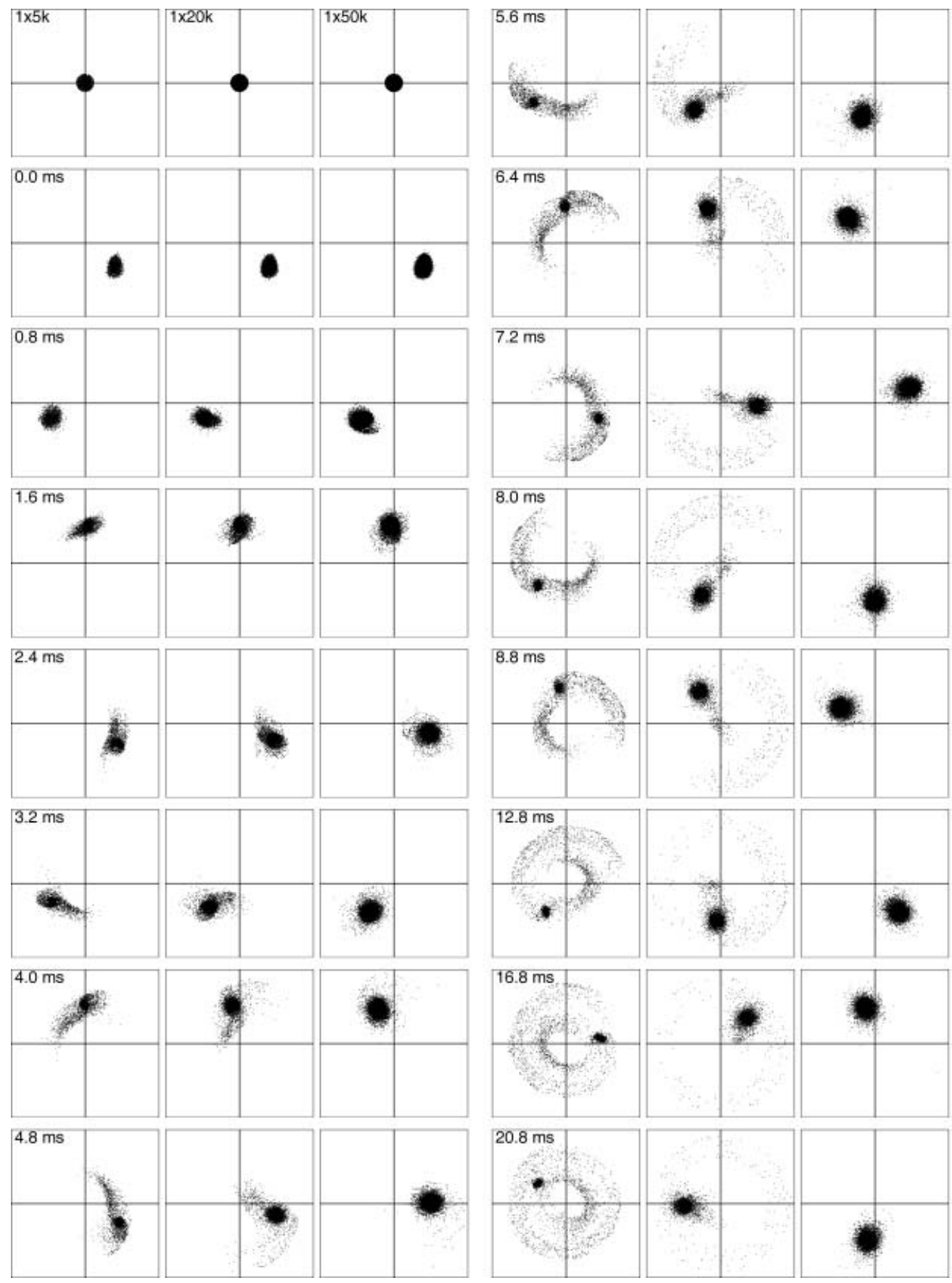

Figure 8. Dynamics of single mass ( $\mathrm{m} / \mathrm{z}$ 100.0) ion clouds (initially $z$-compressed) with 5000 ( $1^{\text {st }}$ and $4^{\text {th }}$ columns), 20000 ( $2^{\text {nd }}$ and $5^{\text {th }}$ columns) and 50000 ( $3^{\text {rd }}$ and $6^{\text {th }}$ columns) ions. Excitation time $45 \mu \mathrm{s}$.

values of excitation time (up to $25 \%$ from initial number for $70 \mu$ s excitation time) and stepwise losses during the comet formation period when the tail particularly reaches the head of the comet. After the comet closes, i.e. when the cloud head reaches its tail, ejection of ions from the cell stops. This behavior is not understood yet and more analysis is required. The majority of lost ions are lost on the side electrodes rather than the trapping electrodes.

\section{Frequency drift during the detection event}

The ion cloud structure in the ICR cell constantly evolves during the detection period. During this time the radial force

Copyright (C) 2007 John Wiley \& Sons, Ltd. acting on a particular ion, which includes coulombic interaction, is changing because of continuous chargedensity redistribution. This in turn leads to effective cyclotron frequency drift during the detection. To follow this drift one should divide the original time-domain signal into parts of short length so that in every interval the ion cloud structure is approximately constant. Then we transform each of the intervals into frequency-domain spectra to determine the evolution of the ion's cyclotron frequency. The fast Fourier transform (FFT) resolution is not high enough for short transients and that is why the covariance algorithm, a parametric spectral estimation method based on minimizing 

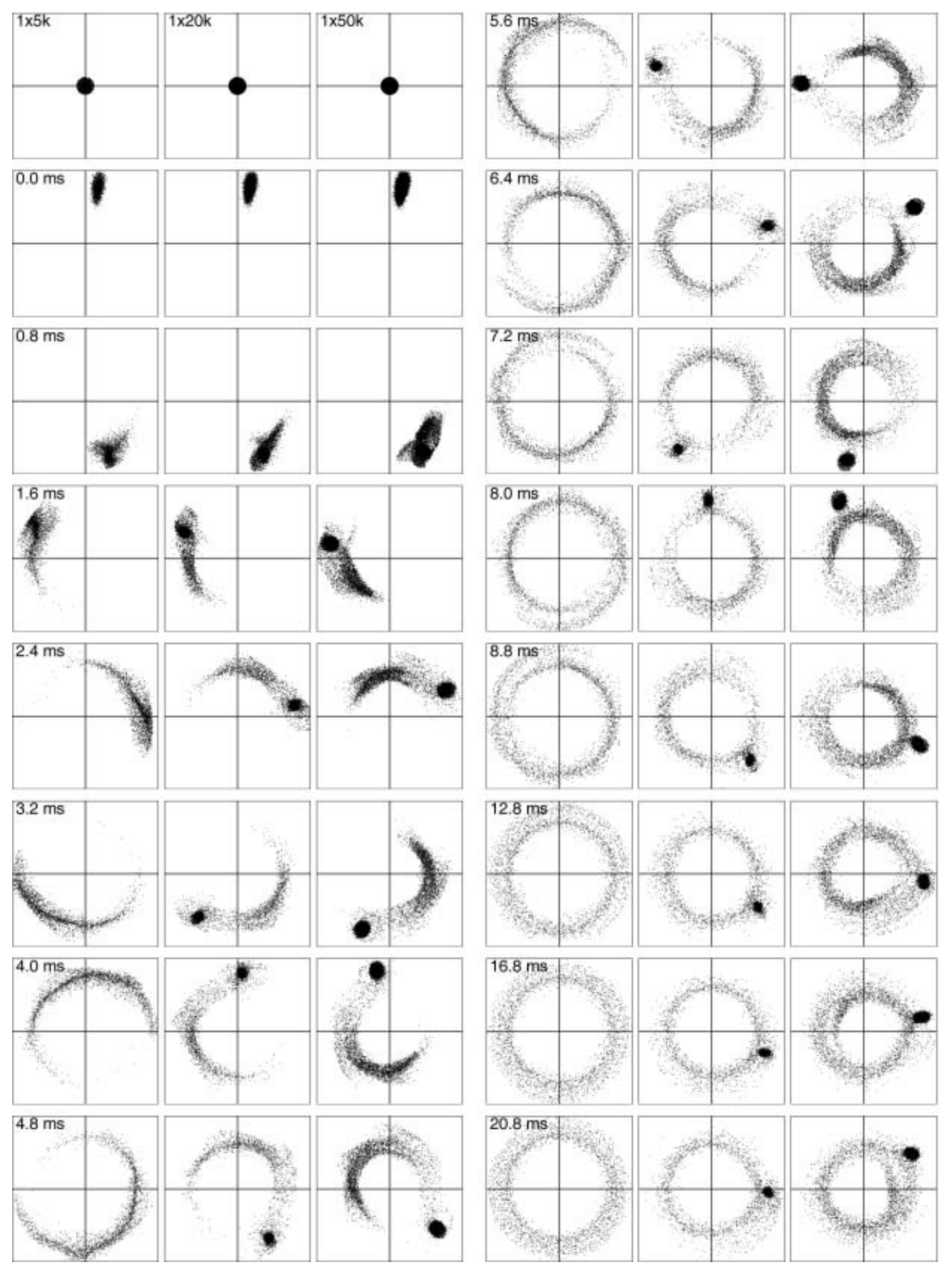

Figure 9. Dynamics of ion clouds (initially z-compressed) with 5000, 20000 and 50000 ions (snapshots layout is the same as in Fig. 10). Excitation time $70 \mu \mathrm{s}$.

the forward prediction error, was used. This method estimates the power spectral density by first estimating the parameters (coefficients) of the linear system that hypothetically 'generate' the signal. It tends to produce better results than classical non-parametric methods when the data length of the available signal is relatively short.

We applied this method to a time-domain signal (3000 000 time-steps $=0.2 \mathrm{~s}$ ) with a relatively long coherent part and sharp decay. The signal was divided into 3000 intervals of equal length (1000 time-steps $=10$ cyclotron periods). For each interval Matlab toolbox function pcov implements the Copyright (C) 2007 John Wiley \& Sons, Ltd. covariance method (order of the model, $p=200$, number of points, $n f f t=4194304)$. In Fig. 11 the results of frequency drift analyses are presented for the signal obtained from an initially $z$-elongated ion cloud (50000 ions, $m / z 100, B=1 \mathrm{~T}$, trapping voltage $1 \mathrm{~V}$, relaxation period $420 \mu \mathrm{s}$ ( $P=1.5 \mathrm{mTorr}$ ), excitation voltage $10 \mathrm{~V}$, excitation period $59.4 \mu \mathrm{s}$, detection period $0.2 \mathrm{~s}$ ). Figure 11(a) shows the time-domain and frequency-domain spectrum corresponding to the first 2 million time-steps $(0-0.13 \mathrm{~s})$ and Fig. 11(b) shows the last million of the time-steps $(0.13-0.2 \mathrm{~s})$. We can see that besides monotonic drift there are spikes on the dependence of frequency on time. As can be seen from Fig. 11(c), where ion 


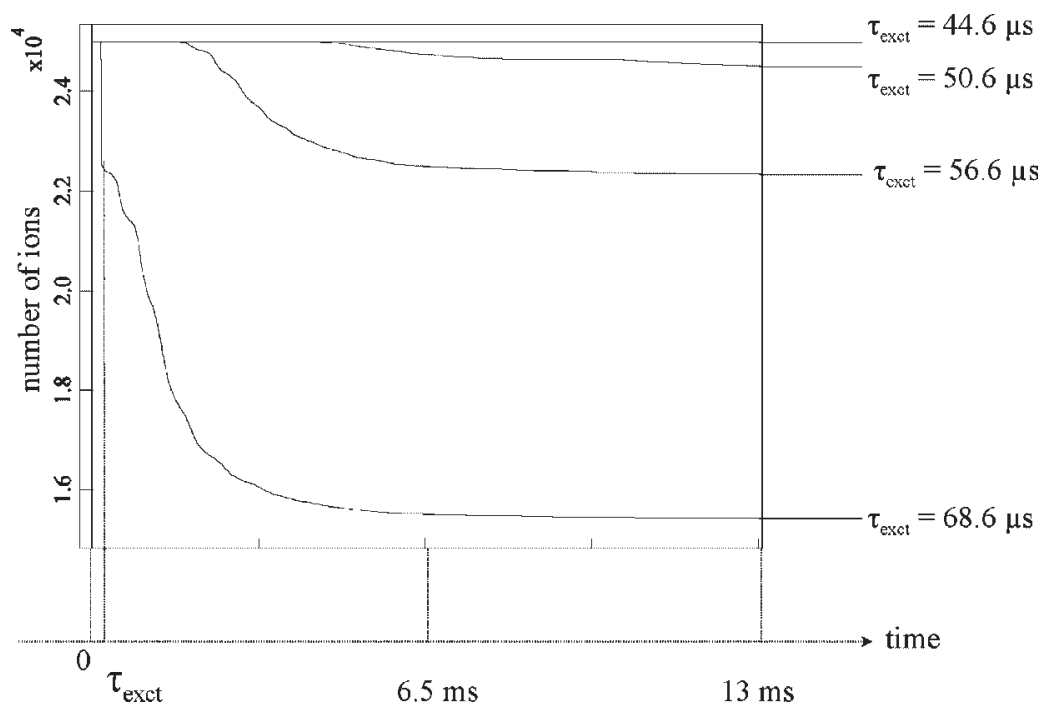

Figure 10. Ion losses during excitation and detection periods. The total number of ions in the cell is presented as a function of time after the start of the RF-excitation event. Up to $25 \%$ from the initial number of ions are lost for 70 us excitation time simulation. After the comet closes, ions ejection from the cell stops.
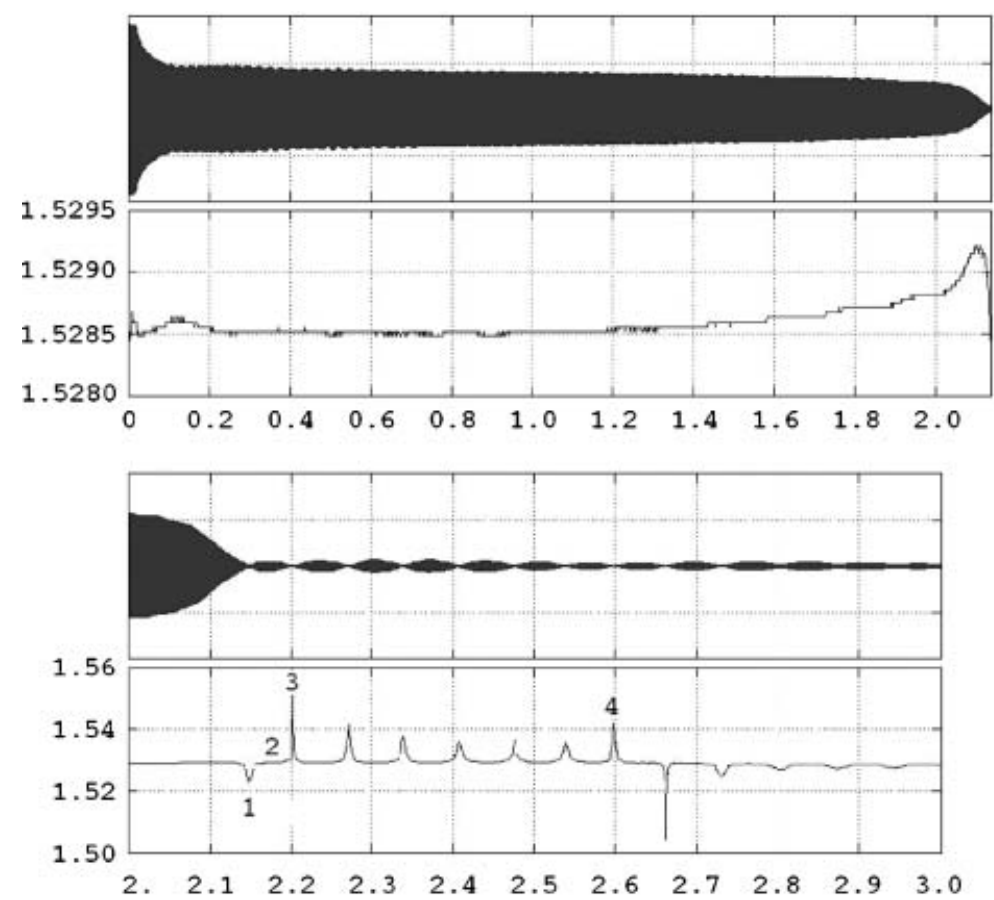

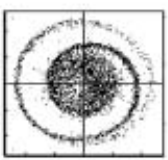

Position 1

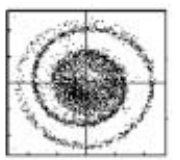

Position 2

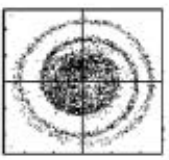

Position 3

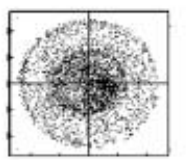

Position 4

Figure 11. The result of frequency drift analysis during the detection event: (a) time-domain and frequency-domain spectrum for the first $0.13 \mathrm{~s}$ of the signal and (b) time-domain and frequency-domain spectrum for the rest of the detection time $0.13-0.2 \mathrm{~s}$. On the frequency-domain spectrum the frequency is given in $10^{5} \mathrm{~Hz}$. In both cases the horizontal axis is scaled in millions of time-steps. Besides monotonic drift there are spikes on the frequency dependence on time. (c) lon density distribution at different positions on the time-scale. For more details, see Supplementary Material. 


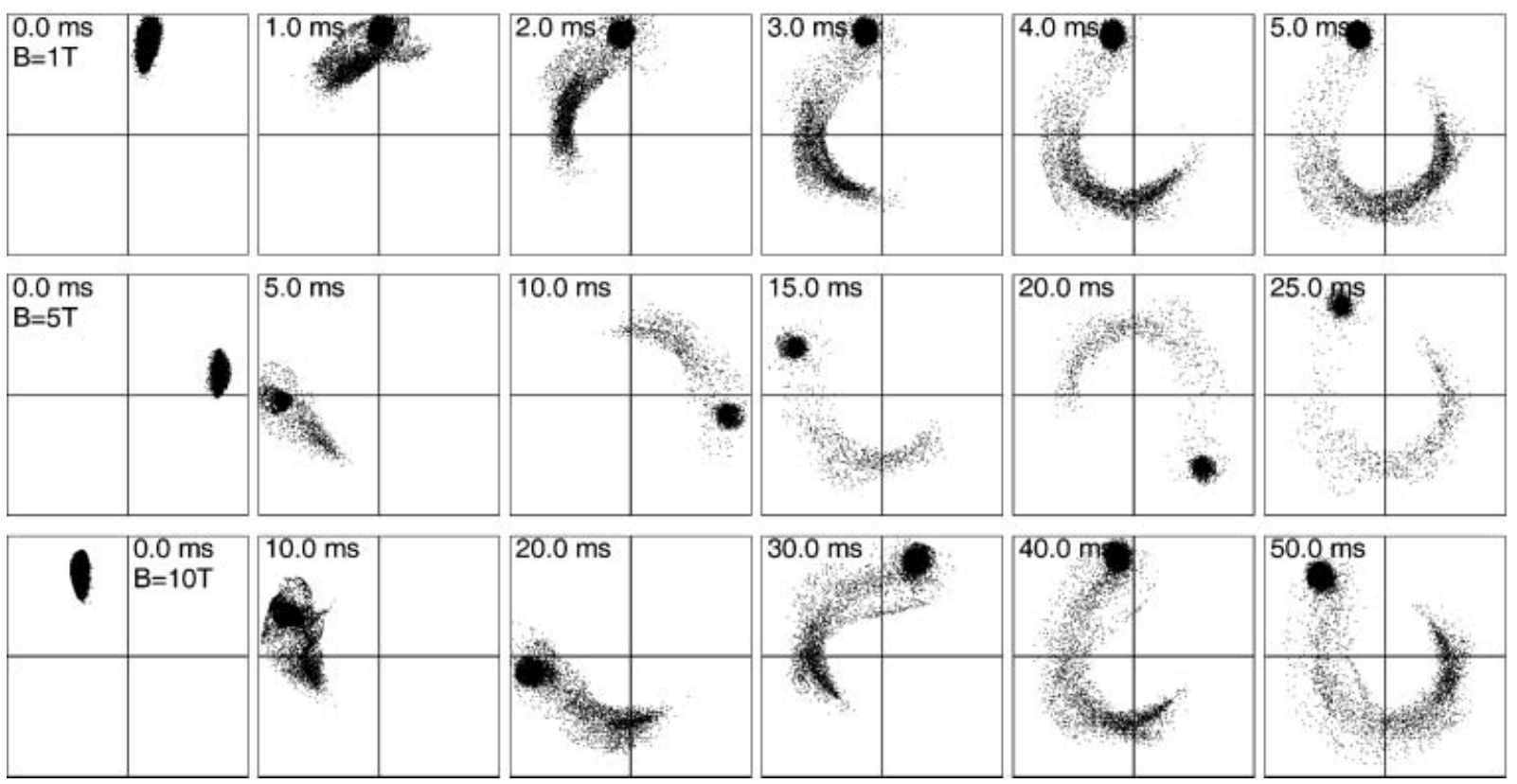

Figure 12. Dependence of ion cloud dynamics on magnetic field strength. Snapshots of ion $x y$ positions for the initially $z$-stressed 50000 ion cloud are shown for three different magnetic fields: 1 T (top), 5T (middle), and 10 T (bottom). Excitation time $70 \mu \mathrm{s}$. The evolution time to reach different stages of cloud evolution is proportional to the magnetic field strength.

distribution snapshots obtained for different detection times are presented, these spikes are caused by charge redistribution inside the cloud having periodical behavior.

\section{Dependence of ion cloud dynamics on magnetic field magnitude}

In Fig. 12 the comparison of ion cloud dynamics for three different magnetic fields is presented. The figure clearly demonstrates that the time to reach different stages of cloud evolution is proportional to the magnetic field magnitude. Note that the increased magnetic field strength does not lead to a change in ion cloud structure but only slows down its evolution. This implies that once the phase-locking phenomenon happens at $1 \mathrm{~T}$ it will also happen at $10 \mathrm{~T}$, but the comet extension will be 10 times slower (in real time) in the latter case. This retardation is due to the linear decrease of magnetron frequency (which is responsible for comet extension rate) with growth of the magnetic field strength. The strength of the magnetic field does not have an influence on the number of ions needed for phase locking to occur.

\section{The behavior of two interacting ion clouds in the ICR cell}

In Fig. 13 the snapshots of two different $m / z(100,100.3)$ ion ensembles during a detection event are presented for different numbers of ions $N$ in the ensembles. We can see that at $N=350000$ the two clouds are moving synchronously. This phenomenon is known as peak coalescence. ${ }^{56}$ In FTICR spectra we see only one peak instead of two with its own $\mathrm{m} / \mathrm{z}$. In Fig. 14 the minimal number of ions in the ensemble needed for peak coalescence to occur is presented as a function of the

Copyright (C) 2007 John Wiley \& Sons, Ltd. magnetic field magnitude. It is clear that this peak coalescence shows the expected quadratic dependence on the magnetic field strength. Note that this coalescence is a different type of motion 'locking' to the phase locking described in the previous paragraph. We should note that the beginning of coalescence with increasing magnetic field strength is difficult to detect precisely. More extensive calculations should be done at higher magnetic field to prove the quadratic dependence on the magnetic field strength.

\section{The behavior of three interacting ion clouds in the ICR cell}

In Fig. 15 the results of modeling the three ion cloud interactions are presented for three totally different numbers of singly charged ions in the ICR cell (75000, 150000 and $225000)$, with the same relative amount of different $\mathrm{m} / \mathrm{z}$ inside the clouds. Ion mass-to-charge ratios in the clouds were chosen to be close to each other $(99.7,100.0$ and 100.3) to observe the effect of peak coalescence. A strong difference in the behavior of all three clouds is evident for total numbers of ions larger than 100 000. During evolution because of strong ion-ion interaction smaller $\mathrm{m} / \mathrm{z}$ ions are acquiring larger cyclotron radius and because of this are forming comet-like structures. Two higher $m / z$ ion clouds $(100.0,100.3)$ coalesce, while the $\mathrm{m} / \mathrm{z} 99.7$ cloud is very unstable and splits into two for the 225000 ions calculation. From FTICR spectra presented in Fig. 16 for all three cases it can be seen that even for the smallest number of ions, 75000, ion-ion interaction between the clouds causes a strong disturbance of the peak magnitudes and positions. 

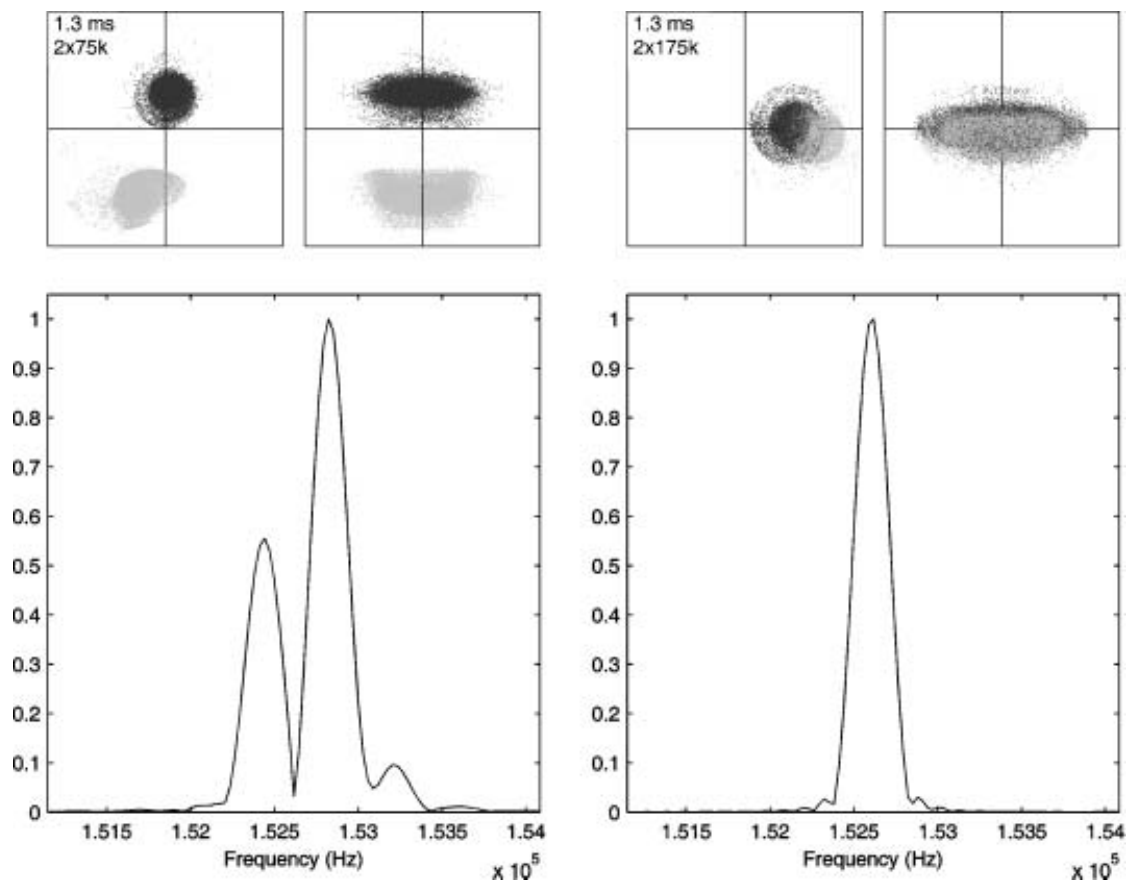

Figure 13. Snapshots in $x y$ and $y z$ perspective of two different $m / z(100.0,100.3)$ ion ensembles during the detection event (after $1.3 \mathrm{~ms}$ ) and corresponding FTICR spectra for 150000 ions (left) and 350000 ions (right) in the ensembles, $B=1 \mathrm{~T}$. The populations of ions with different $m / z$ values are the same in each simulation. At higher number density the clouds are moving synchronously. Corresponding FTICR spectrum displays only one peak instead of two.

\section{DISCUSSION}

As we see from the results the behavior of an ion cloud in an ICR cell depends on many parameters. The main parameter is the number of ions in the cell. At small numbers of ions (around 1000) we see pronounced comet structure formation. The explanation for comet formation is simple. The tail of the comet is formed by ions having larger z-oscillation amplitudes. In an ideal hyperbolic cell the ion effective (cyclotron minus magnetron) frequency is independent of $z$-oscillation amplitude. This is not the case in cubic and cylindrical cells. The larger is the $z$-amplitude the larger is the ion magnetron frequency at turning points (where ions are spending an essential amount of time). There, the non-ideal

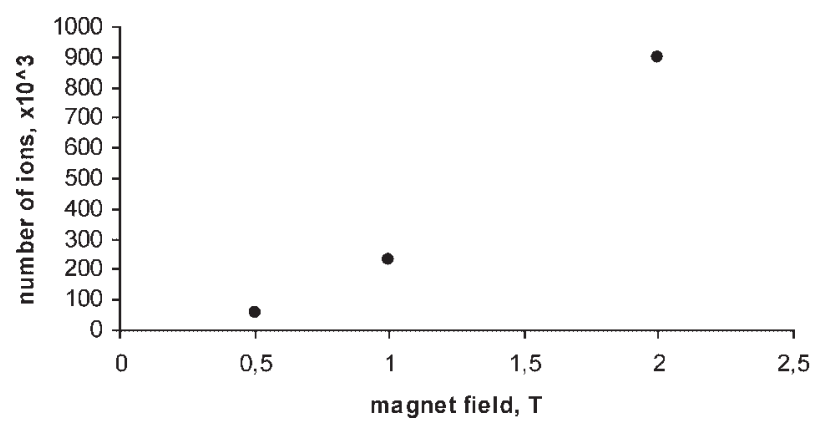

Figure 14. The minimal number of ions in the ensemble needed for peak coalescence to occur is presented as a function of the magnet field strength. The dependence is nearly quadratic.

Copyright (C) 2007 John Wiley \& Sons, Ltd. field results in a smaller rotation frequency of ions with larger $z$-amplitude (that is roughly equal to the difference between the cyclotron and magnetron frequencies). Already a relatively short time after their formation the head of the comet (formed by ions with smaller $z$-amplitude) reaches the tail and the ICR signal disappears. This implies that it is impossible to obtain high resolution at small ion numbers with non-ideal cubic and cylindrical cells. Only in the case of an ideal hyperbolic cell can we avoid this comet structure formation and obtain high resolution even at low ion numbers.

The new phenomenon revealed by our modeling is phase locking or 'condensation' of the same $\mathrm{m} / \mathrm{z}$ ion ensembles. When the number of ions is increasing we see a kind of condensation of part of the ion ensemble with pronounced phase locking. We want to stress here that the phase locking of the same $\mathrm{m} / \mathrm{z}$ ions looks similar to phase locking of close $\mathrm{m} / \mathrm{z}$ ions, which we call coalescence, but in fact it is a different phenomenon. We will investigate this phenomenon more carefully in the future. We see similarity between our observations and observations made in multiple pass TOF instruments, where high resolution is obtained only with a large number of ions and this effect is attributed to their phase locking. ${ }^{57,58}$

The main difference is its dependence on the magnetic field strength. The number of ions in the cell (or better to say charge density) at which the condensation of the same $m / z$ ion clouds starts is independent of magnetic field, while the number of ions necessary for phase locking depends quadratically on magnetic field. Condensation takes place 

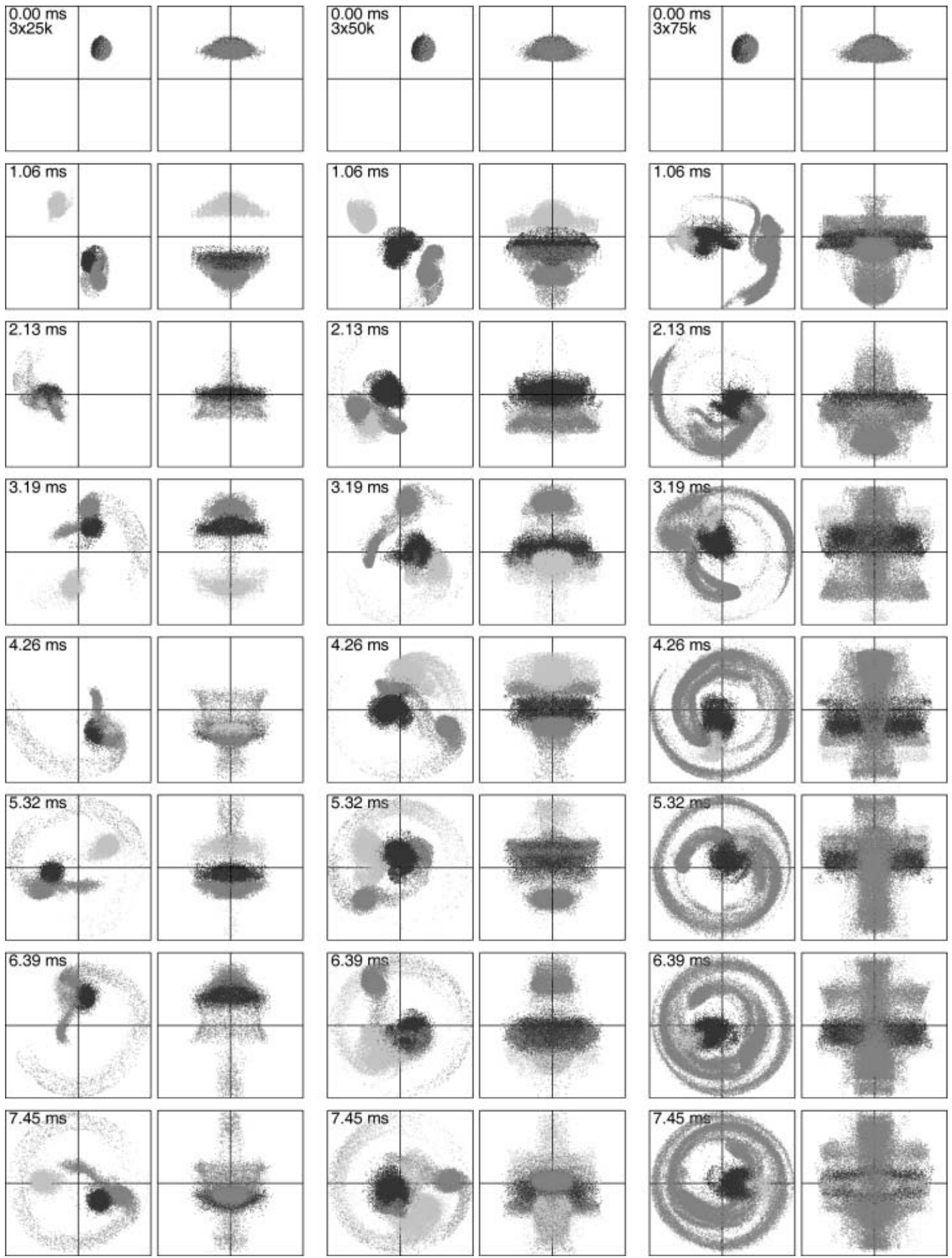

Figure 15. Snapshots in $x y$ and $y z$ projections of three interacting ion clouds $(\mathrm{m} / z 99.7,100.0$, and 100.3) for three different total numbers of ions in the ICR cell: 75000 (left), 150000 (middle) and 225000 (right). The number of ions with different $\mathrm{m} / \mathrm{z}$ values is the same in each simulation. Strong difference in the behavior of all three clouds is evident for total numbers of ions larger than 100000.

in different parts of the ion cloud depending on the initial cloud configuration. For $z$-compressed initial cloud forms we have condensation at the head of the comet and for $z$-elongated at different regions of the comet tail. The condensed part has the form of an ellipsoid. It survives Copyright (C) 2007 John Wiley \& Sons, Ltd. quite a long time giving the main contribution to the ICR signal. After some evolution time this cigar-shaped cloud becomes isolated from the other part of the ion ensemble. In the case of a z-compressed initial cloud the condensed part is positioned outside the cylinder, which is formed by the 

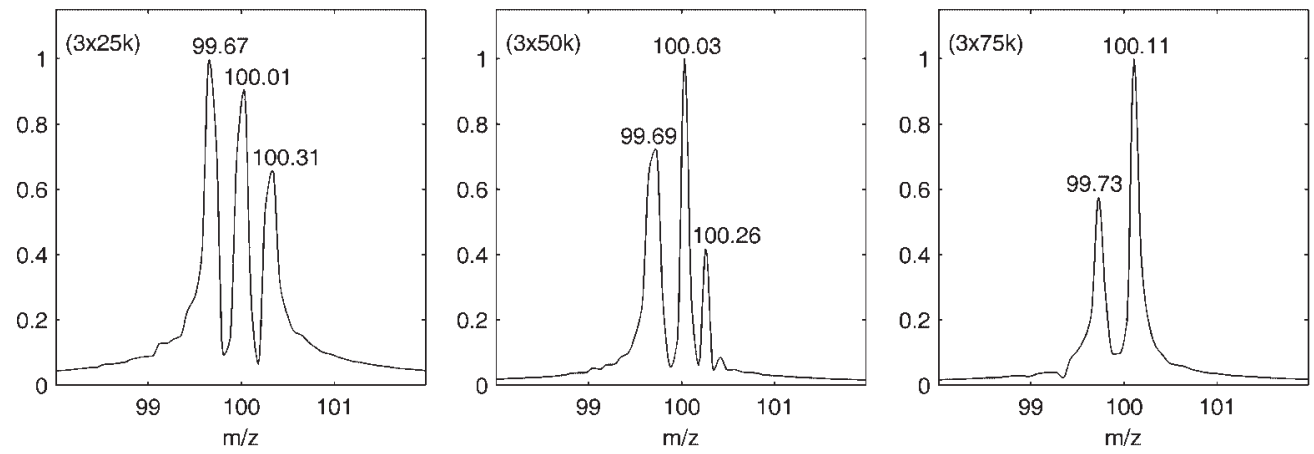

Figure 16. Fourier transform of the time-domain signals induced by ion clouds from Fig. 15.

non-condensed ions and in the case of a $z$-elongated initial cloud it becomes encapsulated into the cylinder of non-condensed ions. This pronounced difference in behavior of initially $z$-compressed and z-elongated ions has the following explanation. The ions in the tail have a smaller rotation radius (and kinetic energy) because they have a larger $z$-oscillation amplitude and are exited to a smaller radius because of excitation field inhomogeneities. At their z-oscillation turning points, the excitation field is weaker compared to the central regions. So if a condensation in the head part of the cloud occurs, this condensed part having higher rotation radius will be outside of the rest of the non-condensed ensemble. If condensation takes place in the tail region the condensed ions will have a smaller radius and will be encapsulated by the rest of ensemble. For these two distinctly different cases there is a big difference in the total coulombic interaction between the ions, which gives the ICR signal, and the rest of the cloud. In the case of a $z$-compressed initial cloud the condensed part of the ion cloud experiences a strong repulsion from the rest of the ion cloud. This repulsion decreases the detected frequency. In the other case, when the condensed part of the cloud is inside a cylinder formed by other ions, we do not have the strong influence of the coulombic forces from this cylinder on the ion frequency and the resulting detected ICR signal frequency. If we increase the number of ions further we will see strong phase locking for all ions in the cloud in the case of single $\mathrm{m} / \mathrm{z}$ ensembles and peak coalescence in FTICR spectra when two or more clouds of different $m / z$ ions are rotating in the cell. The coalescence is a result of the phase locking of ions having different (but close) $\mathrm{m} / \mathrm{z}$ values. The nature of this effect is the same as discussed above for ions of the same $\mathrm{m} / \mathrm{z}$ having different $z$-oscillation amplitudes and as a consequence different effective ICR frequencies. The phase locking and coalescence both take place in the initial ion clouds (before excitation) and survive during the excitation and detection period.

At small excitation times we observe phase locking for smaller numbers of ions. As we see from Fig. 7 the potential profile along the axis has a more pronounced minimum at smaller radius. It is getting flat when the radius increases. So at small excitation times we have higher local ion densities at the same number of ions in the cell.

Phase locking is a crucial effect for FTICR-MS. Without phase locking ions cannot induce a long-lasting signal in

Copyright (C) 2007 John Wiley \& Sons, Ltd. conventional ICR cells (cubic or any kind cylindrical) because the dephasing of their cyclotron motion is in the millisecond range. As a result it is impossible to obtain signals in conventional cells for numbers of ions smaller then $10^{3}$.

The mechanism of ion evaporation from the cloud is not understood yet. It is clear that ion-ion interactions cause a redistribution of the kinetic energy and ions receiving high enough energy are striking the cell electrodes. This is a topic that will be investigated further. The results presented in this paper clearly demonstrate that all analytical considerations undertaken so far were too naïve to describe it. With the next generation of PIC codes currently under development we are going to undertake research concentrated on:

1. SWIFT vs. chirp excitation;

2. Effect of frequency sweep (low-to-high vs. high-to-low);

3. Coalesences - highest $m / z$ at which two equally abundant packets of ions separated by $1 \mathrm{Da}$ in mass, as a function of B-field;

4. Isotopic envelope shape;

5. Non-cubic and other than Penning trap type cells;

6. Effect of inhomogeneous B-field; and

7. Effect of various ion injection modes.

\section{SUPPLEMENTARY MATERIAL}

Supplementary electronic material for this paper is available in Wiley InterScience at: http://www.interscience.wiley. com/jpages/0951-4198/suppmat/

\section{Acknowledgements}

We would like to thank Ms M. S. Sharova (Dept. of Computer Sciences, Moscow State University, Moscow, Russia) for her participation in early stages of the study. This work was financially supported by the "Nederlandse organisatie voor Wetenschappelijk Onderzoek (NWO)" by the Dutch-Russian collaboration grant numbers NOW-RFBR 047-016-022 and RFBR-No. 02-03-32301. The work was partially supported by RFBR-No. 05-07-90238 Grant. Part of this work was carried out in the context of the Virtual Laboratory for e-Science project (http://www.vl-e.nl) which is supported by a BSIK grant from the Dutch Ministry of Education, Culture and Science $(\mathrm{OC} \& W)$ and is part of the ICT innovation program of the Ministry of Economic Affairs (EZ). This work is embedded in the research program of the "Stichting voor Fundamenteel Onderzoek der Materie (FOM)". 


\section{REFERENCES}

1. Huang J, Tiedemann PW, Land DP, McIver RT, Hemminger JC. Int. J. Mass Spectrom. Ion Processes 1994; 134: 11.

2. Naito Y, Inoue M. J. Mass Spectrom. Soc. Jpn. 1994; 42: 1.

3. Mitchell DW, Smith RD. Phys. Rev. E 1995; 52: 4366.

4. Mitchell DW, Smith RD. J. Mass Spectrom. 1996; 31: 771.

5. Mitchell DW, Smith RD. Int. J. Mass Spectrom. Ion Processes 1997; 165/166: 271.

6. Mitchell DW. J. Am. Soc. Mass Spectrom. 1999; 10: 136.

7. Brown LS, Gabrielse G. Rev. Mod. Phys. 1986; 58: 233.

8. Rainville S, Thompson JK, Pritchard DE. Instrumentation and Measurement, IEEE Trans. 2003; 52: 292.

9. Ledford EB Jr, Rempel DL, Gross ML. Anal. Chem. 1984; 56: 2744.

10. Herold LK, Kouzes RT. Int. J. Mass Spectrom. Ion Processes 1990; 96: 275.

11. Hearn BA, Watson CH, Baykut G, Eyler JR. Int. J. Mass Spectrom. Ion Processes 1990; 95: 299.

12. Xiang X, Guan S, Marshall AG. J. Am. Soc. Mass Spectrom. 1994; 5: 238.

13. Guan S, Pasa-Tolic L, Marshall AG, Xiang X. Int. J. Mass Spectrom. Ion Processes 1994; 139: 75.

14. Naito Y, Inoue M. Rapid Commun. Mass Spectrom. 1997; 11: 578.

15. Londry FA, Alfred RL, March RE. J. Am. Soc. Mass Spectrom. 1993; 4 : 687.

16. Julian RK, Nappi M, Weil C, Cooks RG. J. Am. Soc. Mass Spectrom. 1995; 6: 57.

17. Dahl DA. SIMION 3D version 7.0 User's manual, 1995.

18. Dubin DHE, O'Neil TM. Rev. Mod. Phys. 1999; 71: 87.

19. Talroze VL, Nikolaev EN. Adv. Mass Spectrom. 1986; 12: 343.

20. Jeffries JB, Barlow SE, Dunn GH. Int. J. Mass Spectrom. Ion Processes 1983; 54: 169.

21. Gross ML, Rempel DL. Science 1984; 226: 261.

22. Chen SP, Comisarow MB. Rapid Commun. Mass Spectrom. 1992; 6: 1.

23. Gorshkov MV, Marshall AG, Nikolaev EN. J. Am. Soc. Mass Spectrom. 1993; 4: 855.

24. Nikolaev EN, Gorshkov MV. Int. J. Mass Spectrom. Ion Processes 1985; 64: 115.

25. Gorshkov MV, Nikolaev N. Int. J. Mass Spectrom. Ion Processes 1993; 125: 1.

26. Nikolaev EN, Rakov VS, Futrell JH. Int. J. Mass Spectrom. Ion Processes 1996; 157/158: 215.

27. Nikolaev EN, Neronov YI, Gorshkov MV, Tal'roze VL. Pis'ma $v$ Zhurnal Eksperimental'noi i Teoreticheskoi Fiziki 1984; 39: 441.

28. Peurrung AJ, Kouzes RT, Barlow SE. Int. J. Mass Spectrom. Ion Processes 1996; 157/158: 39.

29. Wang TCL, Marshall AG. Int. J. Mass Spectrom. Ion Processes 1986; 68: 287.

30. Uechi GT, Dunbar RC. J. Am. Soc. Mass Spectrom. 1992; 3: 734.

31. Peurrung AJ, Kouzes RT. Int. J. Mass Spectrom. Ion Processes 1995; 145: 139

32. Miluchihin NV, Miura K, Inoue M. Rapid Commun. Mass Spectrom. 1993; 7: 966.

33. Nikolaev EN, Miluchihin NV, Inoue M. Int. J. Mass Spectrom. Ion Processes 1995; 148: 145.

34. Pasa-Tolic L, Huang Y, Guan S, Kim HS, Marshall AG. J. Mass Spectrom. 1995; 30: 825.

35. Anderson JS, Laude DA. Int. J. Mass Spectrom. Ion Processes 1996; 158: 163

36. Stults JT. Anal. Chem. 1997; 69: 1815.

37. Peurrung AJ, Kouzes RT. Phys. Rev. E 1994; 49: 4362.

38. Birdsall CK, Langdon AB. Plasma Physics via Computer Simulation. McGraw-Hill, New York, 1985.

39. Hockney RW, Eastwood JW. Computer Simulation Using Particles. Adam Hilger: New York, 1988.

40. Birdsall CK. IEEE Trans. Plasma Sci. 1991; 19: 65.

41. Comisarow MB, Marshall AG. Chem. Phys. Lett. 1974; 25: 282.

42. Comisarow MB. J. Chem. Phys. 1978; 69: 4097.

43. Marshall AG, Verdun FR. Fourier Transforms in NMR, Optical and Mass Spectrometry: A User's Handbook. Elsevier: Amsterdam, 1990

44. Marshall AG, Grosshans PB. Anal. Chem. 1991; 63: 215A.

45. Asamoto B, Dunbar RC. Analytical Applications of Fourier Transform Ion Cyclotron Resonance Mass Spectrometry. VCH: New York, 1991.
46. Comisarow MB. Adv. Mass Spectrom. 1980; 8: 1698.

47. Comisarow MB. Int. J. Mass Spectrom. Ion Processes 1981; 37: 251.

48. Grosshans PB, Marshall AG. Int. J. Mass Spectrom. Ion Processes 1990; 100: 347.

49. Guan S, Marshall AG. Int. J. Mass Spectrom. Ion Processes 1995; 146/147: 261

50. Shockley J. Appl. Phys. 1938; 9: 635.

51. Shockley J. Am. J. Phys. 1959; 27: 418

52. Dunbar RC. Int. J. Mass Spectrom. Ion Processes 1984; 56(1): 1.

53. Grosshans PB, Shields PG, Marshall AG. J. Chem. Phys. 1991; 94(8): 5341.

54. Boris JP. Relativistic plasma simulation-optimization of a hybrid code. Proc. 4th Conf. Num. Sim. Plasmas, Naval Research Laboratory, Washington DC, 3-67, 2-3 November, 1970.

55. Nikolaev EN. 9th Asilomar Conference on Mass Spectrometry, Trapped Ions: Principle, Instrumentation and Applications, Sep 27-Oct 1, 1992.

56. Marshall AG, Hendrickson CL, Jackson GS. Mass Spectrom. Rev. 1998; 17: 1.

57. Pedersen HB, Strasser D, Ring S, Heber O, Rappaport ML, Rudich Y, Sagi I, Zajfman D. Phys. Rev. Lett. 2001; 87: 055001.

58. Strasser D, Geyer T, Pedersen HB, Heber O, Goldberg S, Amarant B, Diner A, Rudich Y, Sagi I, Rappaport M, Tannor DJ, Zajfman D. Phys. Rev. Letters 2002; 89: 283204.

59. Frigo M, Johnson SJ. Proc. IEEE 2005; 93: 216.

60. Frigo M, Johnson SJ. The FFTW webpage (accessed May 20th, 2007;). Available http://www.fftw.org.

61. The OpenMP web page (accessed May 20th, 2007;). Available http://www.openmp.org.

62. Buneman OJ. Comput. Phys. 1967; 1: 517.

\section{APPENDIX 1}

\section{Charge distribution and field interpolation}

Electric field potential $u(\mathbf{r})$ at point $\mathbf{r}=\{x, y, z\}$ produced by $N_{p}$ particles is defined as:

$$
u(\mathbf{r})=\frac{1}{4 \pi \varepsilon_{0}} \sum_{p=1}^{N_{p}} \frac{q_{p}}{\left|\mathbf{r}-\mathbf{r}_{p}\right|} .
$$

On the other hand, the same potential satisfies Poisson's equation:

$$
\Delta u=-\frac{\rho}{\varepsilon_{0}},
$$

where $\rho$ is the volume charge density expressed via the Dirac $\delta$-function for point charges:

$$
\rho(\mathbf{r})=\sum_{p=1}^{N_{p}} q_{p} \delta\left(x-x_{p}\right) \delta\left(y-y_{p}\right) \delta\left(z-z_{p}\right) .
$$

Thus, the calculation of electric potential is reduced to finding a generalized solution of Poisson's equation (Eqn. (1)) with the source function (2) on a finite grid. To do that, it is necessary to switch from generalized function (2) to mesh function $\rho_{i j k}$. One can implement this using a procedure of smoothing (e.g., averaging). Let us associate:

$$
W_{i j k}(x, y, z)=W_{i}(x) W_{j}(y) W_{k}(z) .
$$

function to each grid point $(i, j, k)$. Weighting multiplier functions take different shapes depending on the chosen method of interpolation. We use the linear interpolation method, where weighting multipliers can be written as:

$$
W_{i}(x)= \begin{cases}1-\left|\frac{x-x_{i}}{h}\right|, & \left|x-x_{i}\right|<h \\ 0, & \left|x-x_{i}\right| \geq h\end{cases}
$$


For smoothing (2) with averaging kernel (3) the following expression was used:

$$
\rho_{i j k}=\int \rho(x, y, z) W_{i j k}(x, y, z) d V,
$$

which gives for $\rho_{i j k}$

$$
\rho_{i j k}=\frac{1}{h^{3}} \sum_{p=1}^{N_{p}} q_{p} W_{i j k}\left(x_{p}, y_{p}, z_{p}\right) .
$$

Then, using mesh function $\rho_{i j k}$, we obtain the electric potential value $u_{i j k}$ at all grid points via Poisson's equation solving method which is described below. Effective force is proportional to electric field strength. The latter is found at all grid points by numerical differentiation of $u_{i j k}$. To interpolate the electric field from mesh points to particles, piecewise linear extension of the mesh functions $E_{i j k}^{x}, E_{i j k}^{y}$ and $E_{i j k}^{z}$ is constructed on the basis of weighting functions (3). We assign a value of the mentioned piecewise-linear extension as the field value at point $\left(x_{p}, y_{p}, z_{p}\right)$ occupied by the particle.

\section{APPENDIX 2}

\section{Three-dimensional Poisson solver}

We used direct the Poisson's equation solver based on three-dimensional (3D) fast Fourier transformation (FFT) library routines. Poisson's equation solver is a crucial part of the particle-in-cell (PIC) code and it requires extensive optimization to achieve a reasonable efficiency. We tried different 3D FFT subprograms and our final choice was a FFT routine provided by the FFTW library. ${ }^{59,60}$ All computations were performed on the shared-memory system IBM eServer pSeries 690 Regatta based on 64-bit IBM Power4 $(1.1 \mathrm{GHz})$ processors running IBM AIX OS. Solving Poisson's equation to double precision on a single processor required $0.0049 \mathrm{~s}$, $0.043 \mathrm{~s}$, and $0.58 \mathrm{~s}$ for $32^{3}, 64^{3}$ and $128^{3}$ grids, correspondingly. Use of four processors decreased times to $0.0023 \mathrm{~s}$, $0.015 \mathrm{~s}$, and $0.31 \mathrm{~s}$, correspondingly. The electric potential $u$ inside the ICR trap T was derived by the solution of Poisson's equation with Dirichlet's boundary conditions:

$$
\left\{\begin{array}{l}
\Delta u=-\rho / \varepsilon_{0}, \quad(x, y, z) \in T \\
\left.u\right|_{\Gamma}=g
\end{array}\right.
$$

where $\varepsilon_{0}=8.85 \times 10^{-12} \mathrm{~F} \mathrm{~m}^{-1}$ was the vacuum permittivity, $g$ was the given potential value on the boundary $\Gamma=\partial \mathrm{T}$, and $\rho$ was the charge density.

For the sake of simplicity, a cubic trap with lateral length $L$ is considered here. A generalization on an arbitrary parallelepiped is obvious. First, if an analytical solution of problem (1) is given in the case of homogeneous boundary conditions $\left.u\right|_{\Gamma}=0$, then a numerical solution is found and, finally, similarities are pointed out in both methods. In conclusion of this appendix, some performance issues are discussed.

\section{Analytical solution in case of homogeneous boundary conditions}

Let us remind ourselves that in some cases the analytical solution of differential problem (1) with homogeneous boundary condition $\left.u\right|_{\Gamma}=0$ may be found as the series expansion on the basis of eigenfunctions of the following eigenvalue problem:

$$
\left\{\begin{array}{l}
\Delta v+\lambda v=0, \quad(x, y, z) \in T \\
\left.v\right|_{\Gamma}=0
\end{array}\right.
$$

In the case of adequate smoothness of functions $u$ and $\rho$ (both functions should at least vanish on the boundary), these functions can be expressed via the following series:

$$
\begin{aligned}
& u=\left(\frac{2}{L}\right)^{3 / 2} \sum_{l, m, n} \hat{u}_{l m n} \sin \frac{\pi l x}{L} \sin \frac{\pi m y}{L} \sin \frac{\pi n z}{L}, \\
& \rho=\left(\frac{2}{L}\right)^{3 / 2} \sum_{l, m, n} \hat{\rho}_{l m n} \sin \frac{\pi l x}{L} \sin \frac{\pi m y}{L} \sin \frac{\pi n z}{L} .
\end{aligned}
$$

Here indices $l, m, n$ vary from 1 to $\infty$. After substitution of the above series expansion into Poisson's equation, a relation between Fourier coefficients $\hat{\rho}_{\text {lmn }}$ of known charge and coefficients $\hat{u}_{l m n}$ of unknown potential can be obtained in the following form:

$$
\hat{u}_{l m n}=\frac{\hat{\rho}_{l m n}}{\varepsilon_{0} \lambda_{l m n}} .
$$

So, the solution of the problem (1) with homogeneous boundary condition $\left.u\right|_{\Gamma}=0$ can be expressed as a series expansion:

$$
u(x, y, z)=\sum_{l, m, n} \frac{\hat{\rho}_{l m n}}{\varepsilon_{0} \lambda_{l m n}} \mu_{l m n}(x, y, z)
$$

where

$$
\lambda_{m n l}=\frac{\pi^{2}}{L^{2}}\left(l^{2}+m^{2}+n^{2}\right)
$$

are eigenvalues and

$$
\mu_{l m n}=\left(\frac{2}{L}\right)^{3 / 2} \sin \frac{\pi l x}{L} \sin \frac{\pi m y}{L} \sin \frac{\pi n z}{L}
$$

are eigenfunctions of the differential eigenvalue problem (2). Fourier coefficients $\hat{\rho}_{l m n}$ can be found from the integral:

$$
\hat{\rho}_{l m n}=\int_{T} \rho \mu_{l m n} d V
$$

\section{Numerical solution}

In order to present a numerical solution to differential problem (1) let us introduce in $T$ a rectangular homogeneous mesh:

$$
\bar{\omega}_{h}=\left\{x_{i j k}=\left(x_{i}=i h, y_{j}=j h, z_{k}=k h\right)\right\} .
$$

Here indices $i, j, k$ vary from 0 to $N, h=L / N$. For the sake of simplicity let us consider $N$ to be one and the same in all dimensions. In the case of different partitioning of each dimension, the above expression changes in an obvious manner. By $\omega_{h}$ we denoted internal, and by $\gamma_{h}$ we denoted boundary nodes of the mesh $\bar{\omega}_{h}$.

We substitute differential Laplacian $\Delta$ by the seven-point finite-difference operator $\Delta_{h}=\Delta_{h, x}+\Delta_{h, y}+\Delta_{h, z}$, which is the sum of finite-difference derivation operators in $x, y$, and $z$ directions, and obtain the second order of approximation: $\Delta u-\Delta_{h} u=O\left(h^{2}\right)$.

Let us find mesh function (vector) $u_{i j k}=u\left(x_{i j k}\right)$ that is defined in the mesh points of $\bar{\omega}_{h}$, and being the solution of the 
following algebraic problem:

$$
\left\{\begin{array}{l}
\Delta_{h} u_{i j k}=-\rho_{i j k} / \varepsilon_{0}, \quad x_{i j k} \in \omega_{h}, \\
u_{i j k}=g_{i j k}, \quad x_{i j k} \in \gamma_{h} .
\end{array}\right.
$$

Here $\rho_{i j k}, g_{i j k}$ are the values of $\rho$ and $g$ functions in the corresponding mesh points. Following the well-known rule, we can substitute inhomogeneous boundary conditions on $\gamma_{h}$ into the right-hand side of the equation. For instance, in the node $x_{122} \in \omega_{h}$ we have:

$$
\frac{u_{022}-2 u_{122}+u_{222}}{h^{2}}+\Delta_{h, y, z} u_{122}=-\frac{\rho_{122}}{\varepsilon_{0}} .
$$

Taking into account that $u_{022}=g_{022}$, we get:

$$
\frac{-2 u_{122}+u_{222}}{h^{2}}+\Delta_{h, y, z} u_{122}=-\frac{\rho_{122}}{\varepsilon_{0}}-\frac{g_{022}}{h^{2}} .
$$

We denoted $\Delta_{h, y, z}=\Delta_{h, y}+\Delta_{h, z}$.

So, without loss of generality, we can consider a zero boundary value problem:

$$
\left\{\begin{array}{l}
\Delta_{h} u_{i j k}=-\rho_{i j k} / \varepsilon_{0}, \quad x_{i j k} \in \omega_{h} \\
u_{i j k}=0, \quad x_{i j k} \in \gamma_{h}
\end{array}\right.
$$

Any mesh function (vector) that is defined on the point set $\omega_{h}$ and vanishing on $\gamma_{h}$ can be expanded on the basis of mesh eigenfunctions (eigenvectors) of the algebraic eigenvalue problem for the operator $\Delta_{h}$ :

$$
\left\{\begin{array}{l}
\Delta_{h} v_{i j k}+\lambda v_{i j k}=0, \quad x_{i j k} \in \omega_{h} \\
v_{i j k}=0, \quad x_{i j k} \in \gamma_{h} .
\end{array}\right.
$$

Mesh functions $u_{i j k}, \rho_{i j k}$ may be represented as finite sums:

$$
\begin{aligned}
& u_{i j k}=\left(\frac{2}{L}\right)^{3 / 2} \sum_{l, m, n} \hat{u}_{l m n} \sin \frac{\pi l i}{N} \sin \frac{\pi m j}{N} \sin \frac{\pi n k}{N}, \\
& \rho_{i j k}=\left(\frac{2}{L}\right)^{3 / 2} \sum_{l, m, n} \hat{\rho}_{l m n} \sin \frac{\pi l i}{N} \sin \frac{\pi m j}{N} \sin \frac{\pi n k}{N} .
\end{aligned}
$$

Here indices $l, m, n$ vary from 1 to $N-1$. Substituting these finite sums into the equation, we can express unknown coefficients $\hat{u}_{l m n}$ via known $\hat{\rho}_{l m n}$ :

$$
\hat{u}_{l m n}=\frac{\hat{\rho}_{l m n}}{\varepsilon_{0} \lambda_{l m n}} .
$$

So, the solution of the boundary problem (7) can be expressed as the finite sum:

$$
u_{i j k}=\sum_{l, m, n} \frac{\hat{\rho}_{l m n}}{\varepsilon_{0} \lambda_{l m n}} \mu_{l m n}\left(x_{i j k}\right)
$$

Here

$$
\lambda_{m n l}=\frac{4}{h^{2}}\left(\sin ^{2} \frac{\pi l}{2 N}+\sin ^{2} \frac{\pi m}{2 N}+\sin ^{2} \frac{\pi n}{2 N}\right)
$$

are eigenvalues and

$$
\mu_{l m n}\left(x_{i j k}\right)=\left(\frac{2}{L}\right)^{3 / 2} \sin \frac{\pi l i}{N} \sin \frac{\pi m j}{N} \sin \frac{\pi n k}{N}
$$

are mesh eigenfunctions (eigenvectors) of the algebraic eigenvalue problem (8). Coefficients $\hat{\rho}_{l m n}$ are found from the dot product (that is defined on the space of mesh functions that are defined on the $\omega_{h}$ and vanishing on $\gamma_{h}$ ) for mesh functions (vectors) $\rho_{i j k}$ and $\mu_{l m n}\left(x_{i j k}\right)$ :

$$
\hat{\rho}_{l m n}=h^{3} \sum_{i, j, k} \rho_{i j k} \mu_{l m n}\left(x_{i j k}\right) .
$$

\section{An analogy between analytical and numerical solutions}

An analogy between analytical and numerical solutions

Generally speaking, numerical solution (9) might be envisioned as analytical solution (3) without residual harmonics from $N$ to $\infty$, or, in other words, as $(N-1)$ th partial sum of series (3) (however, the $(N-1)$ th partial sum of series (3) mismatches the finite sum (9) because (a) we consider homogeneous boundary conditions in the analytical case and inhomogeneous boundary conditions in case of numerical solution, (b) eigenvalues of two problems and formulas for $\hat{\rho}_{l m n}$ coefficients are different). It is necessary to indicate that mesh eigenvectors (11) of algebraic eigenvalue problem (8) are, in fact, the projections (restrictions) of eigenfunctions (5) of differential eigenvalue problem (2) to the space of mesh functions that are defined on the $\omega_{h}$ and vanishing on $\gamma_{h}$; eigenvalues (10) of algebraic problem (8) at limit $h \rightarrow 0(N \rightarrow \infty)$ are transformed into eigenvalues (4) of differential problem (2); formula (12) is actually a numerical method for computing integral (6) (quadratic formula).

\section{Efficiency of the numerical solution}

A direct summation of series (9), (12) requires $O\left(N^{6}\right)$ operations and has no advantage over classic methods of system (7) solution (e.g. Gauss' elimination). Formulas (9), (12) represent discrete Fourier transform (DFT) of the $\hat{u}_{l m n}$ and $\rho_{i j k}$ data sets, respectively. A fast Fourier sum calculation may be performed using the FFT algorithm, which with some restrictions on $N$ (as a rule $N$ must be a power of 2, but not necessarily) requires only $O\left(N^{3} / \log N\right)$ arithmetical operations. There are many FFT program packages available now over the internet. We prefer an open-source library named FFTW being developed by Frigo and Johnson. ${ }^{59,60}$ FFTW employs $O(N \log N)$ algorithms for all sizes, including prime numbers. The current version, FFTW 3.1.2, includes parallel (multi-threaded) transforms for shared-memory systems (including OpenMP parallelism ${ }^{61}$ ). Our computations were performed on supercomputer system IBM eServer pSeries 690 Regatta that is a shared memory multiprocessor machine (SMP, also known as symmetric multiprocessor machine). To take advantage of the shared memory, we used the OpenMP interface $^{61}$ when we wrote a parallel program. In addition, the OpenMP support was enabled when building FFTW. Using a parallel library for the DFT results in a very fast solving of Poisson's equation. This is highly important, because we must solve it at each time-step, and the number of time-steps in a computation can attain several millions. However, it is possible to gain even better results using Hockney's method as a tool for numerical solution of Poisson's equation. Hockney's method is based on a combination of a batch series of 2D Fourier transformations and tridiagonal matrix solving routines. Hockney's method has the same $O\left(N^{3}\right) \log N$ complexity as our current 3D FFT 
method (however, it is worth noting that a series of tridiagonal matrix solving routines require only $O\left(N^{3}\right)$ operations), but this algorithm has greater potential when using parallel computers: 2D Fourier transformations and, then, tridiagonal matrix-solving routines are entirely independent of each other, which gives perfect capabilities for parallelization.

\section{APPENDIX 3}

\section{Algorithm for integration of ion motion equation}

To write an equation of motion in the form of a difference equation, we used the scheme described by Hockney and Eastwood, ${ }^{39}$ and applied to the ICR simulation by Mitchell and Smith. ${ }^{5}$ Let $t^{n}$ be a time level number $n$, and $\tau=t^{n+1}-t^{n}$ the time-step. We are centering the Lorentz force relative to the time level $t^{n}$ by averaging $\mathbf{v}^{n-1}$ and $\mathbf{v}^{n+1}$, what gives us the following recursive equation for ion velocity: ${ }^{38,39,62}$

$$
\mathbf{v}^{n+1}-\mathbf{v}^{n-1}=\frac{2 \tau q}{m}\left(\mathbf{E}^{n}+\left[\frac{\mathbf{v}^{n-1}+\mathbf{v}^{n+1}}{2} \times \mathbf{B}\right]\right)
$$

Ion coordinates are calculated on even time levels while velocities, on odd levels. Time derivatives in an equation of motion are determined using a double time-step. To calculate the electric force, which depends only on coordinates, this scheme is equivalent to a leapfrog scheme. ${ }^{39}$ For calculation of magnetic force, which depends only on velocities, this scheme is equivalent to an implicit time-centered scheme. ${ }^{39}$ With this computational scheme, the digital stability is determined by only the character of drift motion (because electric field is computed explicitly on the time level $n$ ). The scheme is absolutely stable (implicit unconditional stability for velocity) in connection to the fast cyclotron rotation. ${ }^{39}$ When the scheme is written in coordinates, the right-hand side of equations will contain different (by coordinates) components of velocities and magnetic field. This makes it necessary to find a matrix solution to the system of implicit equations to determine velocities on the next time-step. Importantly, it is necessary to separate a slow ion motion related to the electric field and fast motion across the field. To avoid this, we use the algorithm described by Boris ${ }^{54}$ and Buneman, $^{62}$ which takes fewer operations. In this algorithm, electric and magnetic forces are separated in the equation system for calculation of the velocities on the time level $(n+1)$ from velocities on time level $n$. To perform the $\mathbf{E}-\mathbf{B}$ separation, two intermediate variables are introduced, these are velocities $\mathbf{v}^{+}$and $\mathbf{v}^{-}$:

$$
\begin{aligned}
\mathbf{v}^{n-1} & =\mathbf{v}^{-}-\frac{q \mathbf{E}^{n}}{m} \tau, \\
\mathbf{v}^{n+1} & =\mathbf{v}^{+}+\frac{q \mathbf{E}^{n}}{m} \tau .
\end{aligned}
$$

If we substitute these new variables into the equation of motion, we find that the electric field disappears from the equation and it is reduced to an equation for simple rotation of an ion in a magnetic field. The ion energy is conserved in this motion because rotation does not change the absolute velocity. The algorithm for the velocity calculation on the level $(n+1)$ from the velocity on the level $(n-1)$ consists of the three steps:

1. Addition of the half of the electric momentum to $\mathbf{v}^{\mathrm{n}-1}$ :

$$
\mathbf{v}^{-}=\mathbf{v}^{n-1}+\frac{q \mathrm{E}^{n}}{m} \tau
$$

2. Determination of $\mathbf{v}^{+}$by transformation via rotation in accordance with:

$$
\frac{\mathbf{v}^{+}-\mathbf{v}^{-}}{\tau}=\lambda \frac{q}{m}\left[\left(\mathbf{v}^{-}+\mathbf{v}^{+}\right) \times \mathbf{B}\right]
$$

3. Addition of the second half of the electric momentum to $\mathbf{v}^{+}$to obtain $\mathbf{v}^{n+1}$ :

$$
\mathbf{v}^{n+1}=\mathbf{v}^{+}+\frac{q \mathbf{E}^{n}}{m} \tau
$$

During the next step, the implicit equations are solved analytically for the upcoming velocities without introducing any digital errors. The obtained factorized equations constitute a basis of the CYLRAD algorithm invented by Boris. ${ }^{54}$ Calculated frequency of ion motion $\omega_{\mathrm{c}}$ is different from the real one $\Omega_{c}$ when a fixed time-step is used in the calculations. Without a frequency correction, the difference equation gives for the angle of rotation on step $\tau$ :

$$
\theta=2 \arctan \left(\frac{q B}{m} \tau\right)=\omega_{c} \tau\left(1-\frac{\left(\omega_{c} \tau\right)^{2}}{12}+\ldots\right) .
$$

So, for $\omega_{c} \tau<0.35$, the error is approximately $1 \% .{ }^{39}$ To make the integration procedure more accurate, we introduced the correction coefficient $\lambda$ into the integration scheme, ${ }^{38,39}$ equal to:

$$
\lambda=\left(\tan \frac{\Omega_{c} \tau}{2}\right) / \frac{\Omega_{c} \tau}{2} .
$$

\section{APPENDIX 4}

\section{Modeling of ion-neutral collisions}

The main part of our simulations was performed for collision-free cases. We have found that even for the largest number of ions in an ensemble $\left(5 \times 10^{5}\right)$ there is not enough time for relaxation of initial velocity distribution via columbic collisions. If we start from a random space distribution inside the cylinder located in the cell center after $100 \mathrm{~ms}$, we do not see equilibrium elliptic distribution. We see collective oscillations along magnetic field lines with amplitudes corresponding to initial positions of ions in the trapping axial field. Even in the collision-free case, we see a loss of ions by way of ejection from the ICR cell during excitation and detection, and collisions do not influence the phase-locking effect. The deformation of ion cloud shape in the collision-free case is determined by the initial velocity distribution for which we used the Maxwell-Boltzmann law. After excitation, the effective temperature of the ion ensemble is increased due to conversion of potential energy of axial oscillation into kinetic energy; this conversion takes place due to lowering of the trapping potential at larger radius of cyclotron motion. For the most realistic simulations, that is when a neutral bath gas is present, ion-neutral elastic collisions are implemented by using the Monte Carlo algorithm. 5,15,32,38 At a given neutral gas mass, temperature, 
pressure, and hard-sphere cross-section, ion-neutral collisions are accurately treated by this method. At the beginning of every simulation run, a random set of about $10^{4}$ neutral atom collision partner speeds are generated from the Maxwell-Boltzmann distribution and stored in an array. In addition, an array of about $10^{5}$ unit vectors is generated to represent possible directions of neutral atom velocities.

At each time-step in the calculation, the following sequence is performed for each ion next to the particle push stage:

1. A neutral atom velocity is chosen by randomly selecting a speed from the Maxwell-Boltzmann array and multiplying this speed by a random unit vector from the precalculated set.

2. A collisional cross-section, $\sigma$, which is the sum of the hard sphere and Langevin cross-sections, is calculated: ${ }^{15}$

$$
\begin{gathered}
\sigma^{\text {langevin }}=\frac{q}{2 \varepsilon_{0} v_{r}} \sqrt{\frac{\alpha_{l}}{\mu}} \\
\sigma^{\text {hard }}=\frac{\pi d_{\text {ion }}^{2}}{4} \\
\sigma=\sigma^{\text {langevin }}+\sigma^{\text {hard }}
\end{gathered}
$$

where $q$ is the charge on the ion, $\alpha_{l}$ is the electric polarizability of the gas atom (for a helium atom $\left.\alpha_{l}=1.8 \times 10^{-42} \mathrm{~F} \mathrm{~m}^{2}\right), \varepsilon_{0}$ is the permittivity of free space, $\mu$ and $v_{r}$ are the reduced mass and the relative velocity of the collision partners, $d_{i o n}$ is the ion size. (In our simulations we consider $d_{\text {ion }}=1 \mathrm{~nm}$ for a $100 \mathrm{Da}$ ion, neutral atoms being point objects.)

3. The probability $P_{c}$ of a collision per time-step $\Delta \tau$ is determined: ${ }^{38}$

$$
P_{c}=1-\exp \left(-n_{g} \sigma v_{r} \Delta \tau\right)
$$

where $n_{g}$ is the neutral gas density.

4. A random number between 0 and 1 is generated. If this number is less than or equal to the probability $P_{c}$, then a collision is deemed to have occurred, in which case:

a. The velocity of the center of mass of the collision partners is determined.

b. The magnitude of the velocity of the ion in the center of mass reference frame is calculated.

c. The particle's center of mass velocity is given a random direction in the center of mass frame (isotropic scattering).

d. The scattered ion's new velocity is transformed to the laboratory reference frame.

\section{APPENDIX 5}

\section{Influence of the computational mesh size on the accuracy of simulations}

In Fig. 17, the influence of the computational mesh size on the accuracy of simulations is shown. Starting from the mesh
$4 \times 4 \times 4$ further increase in number of nodes does not lead to significant changes in ion cloud structure.

\section{APPENDIX 6}

\section{FTICR signal detection}

In accordance with Gauss' law applied to the small element of the electrode surface, the internal normal component of the electric field is defined as $E_{n}=\sigma / \varepsilon_{0}$. The total charge is computed by integrating over the detector plate when the induced surface charge density $\sigma$ is known:

$$
q=\int \sigma d S
$$

After solving Poisson's equation, we calculate the electric potential on all internal mesh points (boundary values are given). Numerical differentiation methods are subsequently applied to compute the electric field at the grid points: a central difference derivation with a second-order approximation is used for internal points, and one-sided first-order differences are used for the boundary points. For example, the $y$ component of the $\mathbf{E}$ field at $(i, j, k)$ point is defined by

$$
\begin{aligned}
E_{i, j, k}^{y} & =\frac{u_{i, j+1, k}-u_{i, j-1, k}}{2 h}, \\
E_{i, 0, k}^{y} & =\frac{u_{i, 1, k}-u_{i, 0, k}}{h}, \\
E_{i, N, k}^{y} & =\frac{u_{i, N, k}-u_{i, N-1, k}}{h}
\end{aligned}
$$

for internal, left and right boundary points, respectively, where $u_{i j k}$ is the potential at the mesh point $(i, j, k)$. However, we may apply formulae with a formally larger order of approximation, e.g.:

$$
E_{i, 0, k}^{y}=\frac{-3 u_{i, 0, k}+4 u_{i, 1, k}-u_{i, 2, k}}{2 h}
$$

gives us the second order on $h$ for mesh point $(i, 0, k)$ to compute the electric field strength at the boundary points, but it may result in significant errors due to peculiarities of the one-sided difference derivation operation.

With the normal components of the electric field at all detection electrode mesh points, it is possible to apply numerical integration to compute the values given by expression (1). We use a two-dimensional analogue of the rectangular quadratic formula for this purpose:

$$
q=\varepsilon_{0} \frac{d^{2}}{N^{2}} \sum_{i=1}^{N-1} \sum_{k=1}^{N-1} E_{i, j, k}^{x} .
$$

Here $j$ is equal to 0 and $N$, where $N+1$ is the number of grid points on the detection electrode along one of the directions and $d$ is the size of the electrode (the signal is picked up from the plates that are parallel to the magnetic field (z-axis)). As a result of this procedure (performed at each time-step), the time-dependent image charge difference between detector plates can be obtained. We apply Fourier transformation on the time-domain signal or its time derivative (which is computed via difference derivations) to obtain the mass spectrum. 

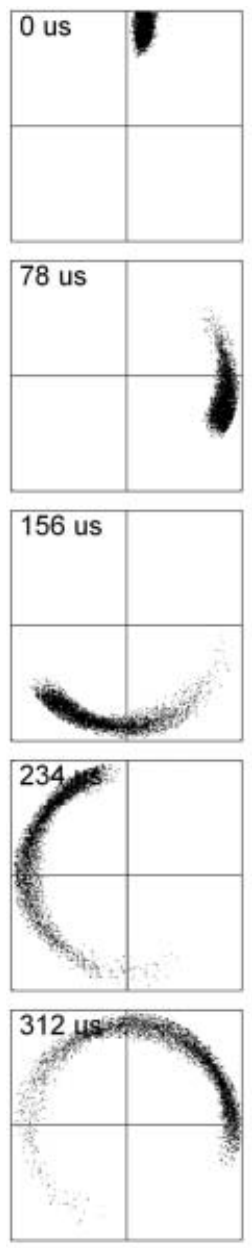

$4 \times 4 \times 4$
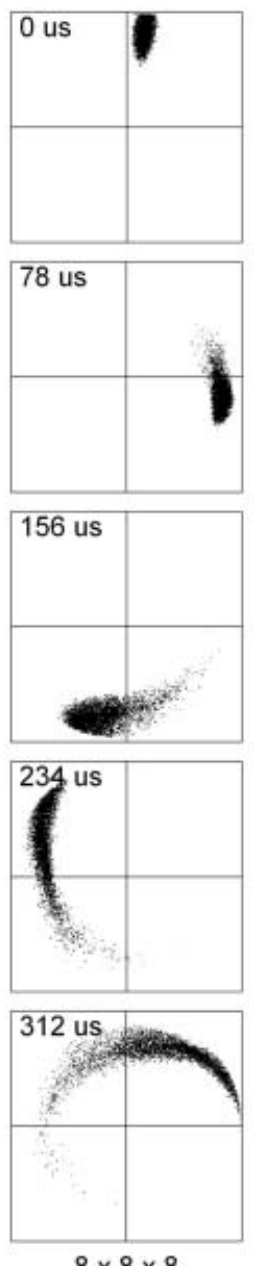
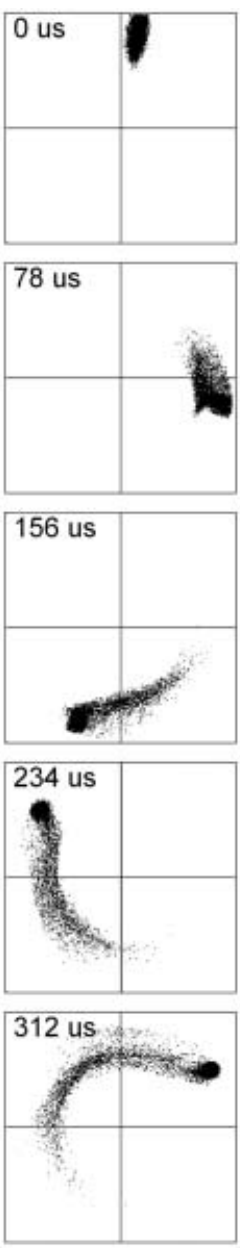

$16 \times 16 \times 16$
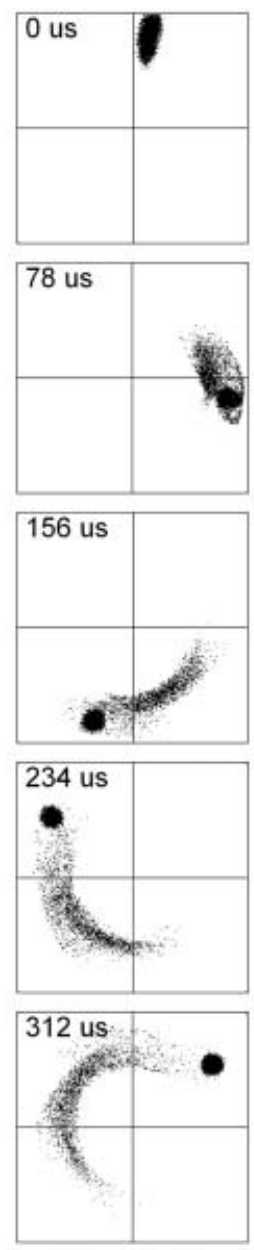

$32 \times 32 \times 32$
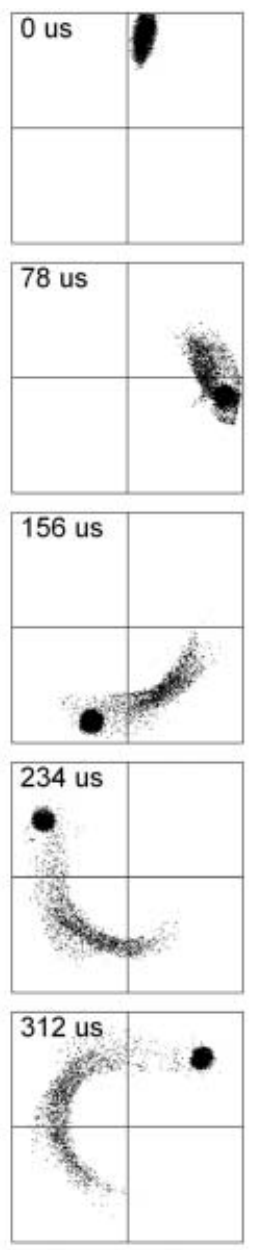

$64 \times 64 \times 64$
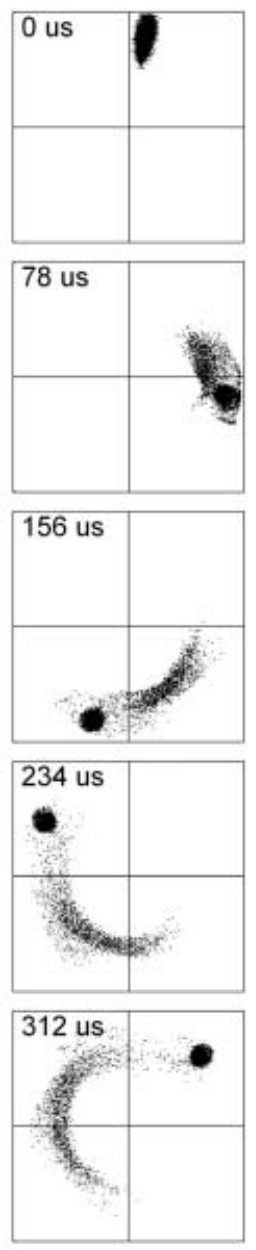

$128 \times 128 \times 128$

Figure 17. Influence of the computational mesh size $\left(4^{3}\right.$ to $128^{3}$, from left to right) on the simulation results for single $\mathrm{m} / \mathrm{z}$ ion cloud. 